OPEN ACCESS

Edited by:

Yu Saida,

Niigata University, Japan

Reviewed by:

Mariana Lazarini,

Federal University of São Paulo, Brazi André Zelanis,

Federal University of São Paulo, Brazi

*Correspondence: Gang Wang wangg@xzhmu.edu.cn Junnian Zheng

jnzheng@xzhmu.edu.cn

${ }^{t}$ These authors have contributed equally to this work

Specialty section:

This article was submitted to Molecular and Cellular Oncology, a section of the journal Frontiers in Oncology

Received: 29 August 2021 Accepted: 03 November 2021 Published: 19 November 2021

Citation:

Chen Y, Han L, Qiu X, Wang G and Zheng J (2021) Neutrophil Extracellular

Traps in Digestive Cancers:

Warrior or Accomplice.

Front. Oncol. 11:766636. doi: 10.3389/fonc.2021.766636

\section{Neutrophil Extracellular Traps in Digestive Cancers: Warrior or Accomplice}

\author{
Yuxin Chen ${ }^{1,2 \dagger}$, Lulu Han ${ }^{1,2,3 \dagger}$, Xiaoyan Qiu ${ }^{1,2}$, Gang Wang ${ }^{1,2,3 *}$ and Junnian Zheng ${ }^{2,3 *}$ \\ ${ }^{1}$ Cancer Institute, Xuzhou Medical University, Xuzhou, China, ${ }^{2}$ Jiangsu Center for the Collaboration and Innovation of Cancer \\ Biotherapy, Cancer Institute, Xuzhou Medical University, Xuzhou, China, ${ }^{3}$ Center of Clinical Oncology, The Affiliated Hospital \\ of Xuzhou Medical University, Xuzhou, China
}

Characterized as a complex of extracellular DNA fibers and granule proteins, neutrophil extracellular traps (NETs) are generated specifically by neutrophils which play a critical role in host defense and immune regulation. NETs have been initially found crucial for neutrophil anti-microbial function. Recent studies suggest that NETs are involved in tumorigenesis and cancer progression. However, the function of NETs in cancer remains unclear, which might be due to the variation of research models and the heterogeneity of cancers. Although most of malignant tumors have similar biological behaviors, significant differences indeed exist in various systems. Malignant tumors of the digestive system cause the most incidence and mortality of cancer worldwide. In this review, we would focus on research developments on NETs in digestive cancers to provide insights on their role in digestive cancer progression and future research directions.

Keywords: neutrophil extracellular trap, cancer, digestive system, immunity, targeted therapy

\section{INTRODUCTION}

As a subset of innate immune cells, neutrophils play a critical role in host defense against microbial infection, and are traditionally recognized as a short-life and terminally differentiated cell (1). Thus, previous studies on neutrophil function on the tumor are limited. However, an increasing number of studies have shown the association of neutrophils with cancer progression, although the roles of neutrophils in cancer progression remain a controversial issue, which might be due to the spatialtemporal tumor microenvironment (2). One of the ways neutrophils participate in cancer progression is through NETosis, wherein neutrophils expel their genomic DNA decorated with proteins (3).

Neutrophil extracellular traps (NETs) are the products of NETosis and were first described by Brinkmann et al. (4) as an extracellular fiber formed by granule proteins and chromatin of neutrophils to capture and kill invading bacteria; NETs were later found to be involved in host immune defense against fungi (5), parasites (6) and viruses (7). However, dysregulated NETs cause the pathogenesis of immune-related diseases. Recently, NETs have been reported to be associated with the immunothrombosis and disease severity caused by COVID-19 $(8,9)$ and considered as a prognostic marker (10) and therapeutic target (11). Studies have shown that NETs play a distinct role in cancer. On the one hand, NETs exert their anti-tumor function by directly killing cancer cells 
or indirectly cooperating with other immune cells. On the other hand, NETs have been found to contribute to cancer progression in several ways, such as inhibiting apoptosis and inducing tumor angiogenesis (12). Although numerous studies have shown that NETs may be more inclined to promote tumor progression, the actual role of NETs in cancer remains unknown, mainly due to the diversity of NET-related components, the complexity of the tumor immune microenvironment, and the differences of various tissues and organs.

The digestive system is composed of the oral cavity, esophagus, stomach, small intestine, colorectum, liver, and pancreas; the malignancy of these components has severely damaged public health (13). According to the global cancer statistics for 2018, the incidence and mortality of digestive cancers approximately account for $29 \%$ and $30 \%$ in all types of cancers worldwide, respectively; these statistics indicate that digestive cancers are not only the most common cancer but also the most frequent cause of cancer-related death all over the world. Among the digestive cancers, colon cancer and gastric cancer represent the top incidence and mortality, respectively (14). Neutrophils have been demonstrated to play a vital role in digestive cancer progression (15). Neutrophil-to-lymphocyte ratio (NLR) is negatively associated with the prognosis of digestive cancers, such as oral squamous cell carcinoma (OSCC) (16), esophageal cancer (17), and gastrointestinal malignancies (18). NETs, acting as a weapon of neutrophils, have also been found to be involved in the progression of digestive cancers (19).

In this review, the formation and degradation of NETs are concisely described first. Second, the relationship between NETs and cancer is briefly summarized from the aspects of tumor growth, metastasis, angiogenesis, and thrombus. the effect of NETs on digestive cancers is analyzed in the third part. Lastly, the possible targeting strategies and future research directions on NETs for cancer therapy are discussed and predicted.

\section{FORMATION AND DEGRADATION OF NETS}

The process of NET formation is defined as NETosis. Upon stimulation, two different ways for NETosis have been proposed, namely, suicidal NETosis and vital NETosis (non-lytic NETosis) (20). In the suicidal approach, decondensed chromatin in active neutrophil is expelled via nuclear envelope and plasma membrane rupture followed by neutrophil death (21), whereas non-lytic NETosis is achieved through the rapid release of NETs components and the reservation of the neutrophil's phagocytic function (20-22). Most of the existing studies have focused on the understanding and effect of NETosis compared with the nonlytic NETosis, which helps in illustrating the nature of NETs.

Regardless of how NETs are formed, the critical process is chromatin decondensation, which is mainly triggered by reactive oxygen species (ROS) and peptidylarginine deiminase 4 (PAD4). Induced by stimulus, such as phorbol myristate acetate and lipopolysaccharide (LPS) (23), nicotinamide adenine dinucleotide phosphate (NADPH)-mediated ROS production leads to the activation and nucleus translocation of myeloperoxidase (MPO) and neutrophil elastase (NE) (24), which subsequently promotes the degradation of specific histones and chromatin decondensation. Moreover, other stimuli, including immune complexes, ionomycin, and nicotine, could induce formation of NETs via mitochondrial ROS independently of NADPH oxidase (25-27). Although these reports suggest that ROS plays a crucial role in the release of NETs, NET formation can transpire without ROS production. Non-lytic NETosis has been reported to be independent from ROS production (22).

Another nuclear chromatin protein, DEK, has been reported to be involved in NETosis. NETosis is restricted in DEK-deficient neutrophils and can be subsequently rescued by recombinant DEK protein; this finding suggests that DEK is crucial in NETs formation (28). Chromatin depolymerization, another critical step for NET formation, depends on histone deamination or citrullination, which is driven by the ribozyme PAD4 $(29,30)$. A recent study shows that PAD4 inhibitors prevent NET formation in sepsis and tumor models $(31,32)$, and PAD4-deficient neutrophils lose the ability to release NETs after stimulation with LPS and tumor necrosis factor (33).

The mechanism of NETs degradation requires further exploration. Studies have shown that during infection, NETs can last for several days (34) and can be gradually degraded and cleared by the nuclease DNase I (35). DNase I injection can rapidly degrade NET-related DNA, while NET-related proteins still exist after DNA degradation (31). DNase I has been found to promote the phagocytosis of NETs by macrophages in vitro, suggesting that the phagocytosis of macrophages may be one of the mechanisms for clearing NETs (36).

Notably, the specific characteristics of NETs, such as mechanisms of formation and degradation, and their exact components, have not been fully elucidated, hampering NETs evaluation in the laboratory setting. NETs can be considered as a type of DNA-protein complex, therefore, several markers have been used to detect the level of NETs at the laboratory, such as cell-free DNA (cfDNA), MPO, NE, citrullinated histone $\mathrm{H} 3$ (citH3), citH3-DNA and MPO-DNA (10, 27, 37, 38). For example, the levels of cfDNA and MPO-DNA are usually employed to measure the NET formation in the serum, plasma or neutrophil culture medium. The protein components of NETs, including MPO, NE, and $\mathrm{CitH3}$, can also be used to determine NET formation in tissue samples by immunohistochemistry and immunofluorescence. However, the golden standard to detect NETs has not yet been illustrated. Therefore, the use of combined markers to evaluate NET formation is reasonable.

\section{FACTORS OF TUMOR-INDUCED NET FORMATION}

NETs have been found in the primary tumor, metastasis, or circulating system in the context of malignant tumors $(37,39,40)$. 
The effect of NETs in cancer was first presented in 2012 (41), and the finding on NETs in cancer tissue was demonstrated in 2013 (42). Several factors, such as granulocyte colony-stimulating factor (G-CSF) (43) and interleukin 8 (IL-8) (44), were also found to stimulate NETs formation in cancer. However, the mechanisms of tumor-induced formation of NETs remain unclear.

Three major aspects may affect NET formation in the presence of cancers. One is the tumor cell itself, which may be the primary factor to affect NETs formation. IL-8, also known as CXCL8, is a major chemokine that promotes the infiltration of polymorphonuclear leukocytes into tissue (45); IL-8 has also been found to be involved in cancer progression (46). Numerous studies confirm that neutrophils are prone to release NETs under the induction of IL-8 in solid and lymphoid-hematopoietic system tumors $(44,47-54)$. G-CSF has been used to increase circulating neutrophils to prevent neutropenia-associated serious infections after systematic chemotherapy (55). However, reports have shown that endogenous G-CSF promotes the progress of several types of cancers, such as neuroblastoma and breast cancer $(56,57)$, and tumor-derived G-CSF plays a critical role in NET formation in breast cancer and lung cancer $(39,41,58)$. Tumor cells can also induce NET formation by secreting a variety of other stimulating factors, such as exosome, transforming growth factor- $\beta$ (TGF- $\beta$ ), interleukin-1 $\beta$ (IL-1 $\beta$ ), extracellular RNA (exRNA), mitochondrial DNA (mtDNA), high mobility group box-1 (HMGB1), cathepsin C (CTSC), tissue inhibitor of metalloproteinases-1 (TIMP-1), CXCL5 (59-69).

Stromal cells in the tumor microenvironment (TME), such as immune cells, endothelial cells, fibroblasts, and adipocytes, also contribute to NET formation (70, 71). Murine lung epithelial-12 cells, activated by Lewis lung cancer cells, provoke NETs release by producing IL-1 $\beta$ (63). IL-17, a cytokine secreted by TH17 cells, has been found to induce NET formation in pancreatic ductal adenocarcinoma (PDAC) (72). Inorganic polyphosphate, a natural molecule, also has been recently found to be a stimulus for NETs formation (73). Mast cells in colon cancer, instead of non-malignant conditions, have been found to generate inorganic polyphosphate, which may promote NETs formation in colon cancer (74). Numerous studies have been conducted on the role of platelets in NET formation, considering that platelets are closely related to the development and progression of cancer (75). Research has shown that activated platelets in pancreatic cancer promote tumor-associated neutrophils to form NETs (76). Another study further demonstrated that activated platelets induce NETs formation via secreting HMGB1 (77). Moreover, HMGB1 is identified as one of the components in NETs (78), which suggests that HMGB1 released by neutrophils may encourage other neutrophils to form NETs. In pancreatic cancer and melanoma, amyloid $\beta$, derived from cancerassociated fibroblasts, facilitates NETs formation via CD11b in a ROS-dependent manner (79). Aside from the abovementioned stimuli, complement 3a (C3a), a type of complement, has also been found to promote the formation of NETs (80).

The third type of factor that induces NET formation is metabolic factors. Plasma redox imbalance is found to encourage NET formation in lung cancer (81). Free fatty acid was discovered to be a stimulus of NET formation in nonalcoholic steatohepatitis, and NETs promote the occurrence and development of hepatocellular carcinoma (82). Although studies have shown that tumors can induce neutrophils to excrete DNA and protein complexes to form NETs, only a few studies have explored the factors that induce NET formation in tumors. Whether other cells or immune components are involved in the regulation of NETs in TME requires further research. In particular, only a few studies have explored the mechanisms by which these stimulus factors lead to increased formation of NETs. Thus, future studies may need to focus on the underlying mechanisms of induction factors. Table 1 collected specific information about inducer factors that promote NET formation in tumors.

\section{FUNCTION OF NETS ON CANCER PROGRESSION}

Studies on the role of NETs in tumors are still limited. Acting as an arm of neutrophils, NETs have been demonstrated to perform an anti-tumor or a pro-tumor function on cancer progression. NETs induced indirectly by retinoic acid exert an anti-tumor function by enhancing the cytotoxicity of neutrophils to breast cancer (83), and Bacillus Calmette-Guerin-NETs inhibit the proliferation and metastasis of bladder cancer (84). NETs can also induce the apoptosis of colon cancer cells to suppress cell proliferation in vitro (85). CD16 ${ }^{\text {high }}$ CD62 $\mathrm{L}^{\mathrm{dim}}$ neutrophils, a subset of neutrophils, is characterized by high expression of CD11b and CD18; NETs derived from these neutrophils have been demonstrated to play an anti-tumor role in head and neck squamous cell carcinoma (86). Recent studies have shown that NETs are correlated with better outcome of ovarian cancer patients (87) and may play an antineoplastic role by inducing the necrosis of melanoma cells (88). As compared with limited anti-tumor reports, several studies provide contrasting results suggesting that NETs mainly play a role in promoting tumor, which is supported by clinical data (89-91).

NETs have been demonstrated to be involved in the entire process of tumor progression, including tumor cell proliferation, adhesion, migration, and metastasis in several in vitro and in vivo models. Moreover, NETs have also been demonstrated to suppress the anti-tumor immune response and to promote cancer-associated thrombosis formation. In addition to directly affecting tumor cells, NETs also contribute to tumor-associated kidney injury in mice (92) and are related to cancer-associated stroke and disseminated intravascular coagulation $(93,94)$. These cancer-related vascular and organ complications may be partly due to vascular dysfunction induced by NETs. Endothelial cells are forced to undergo morphological changes under the influence of NETs, including retraction from cell-cell junctions and a more pro-coagulative phenotype (95). The vascular functions of organs have also been found to be affected by NETs instead of tumor cells themselves $(43,96)$. However, only a few studies have been conducted on the specific 
TABLE 1 | Detailed information about NETs induced by tumors.

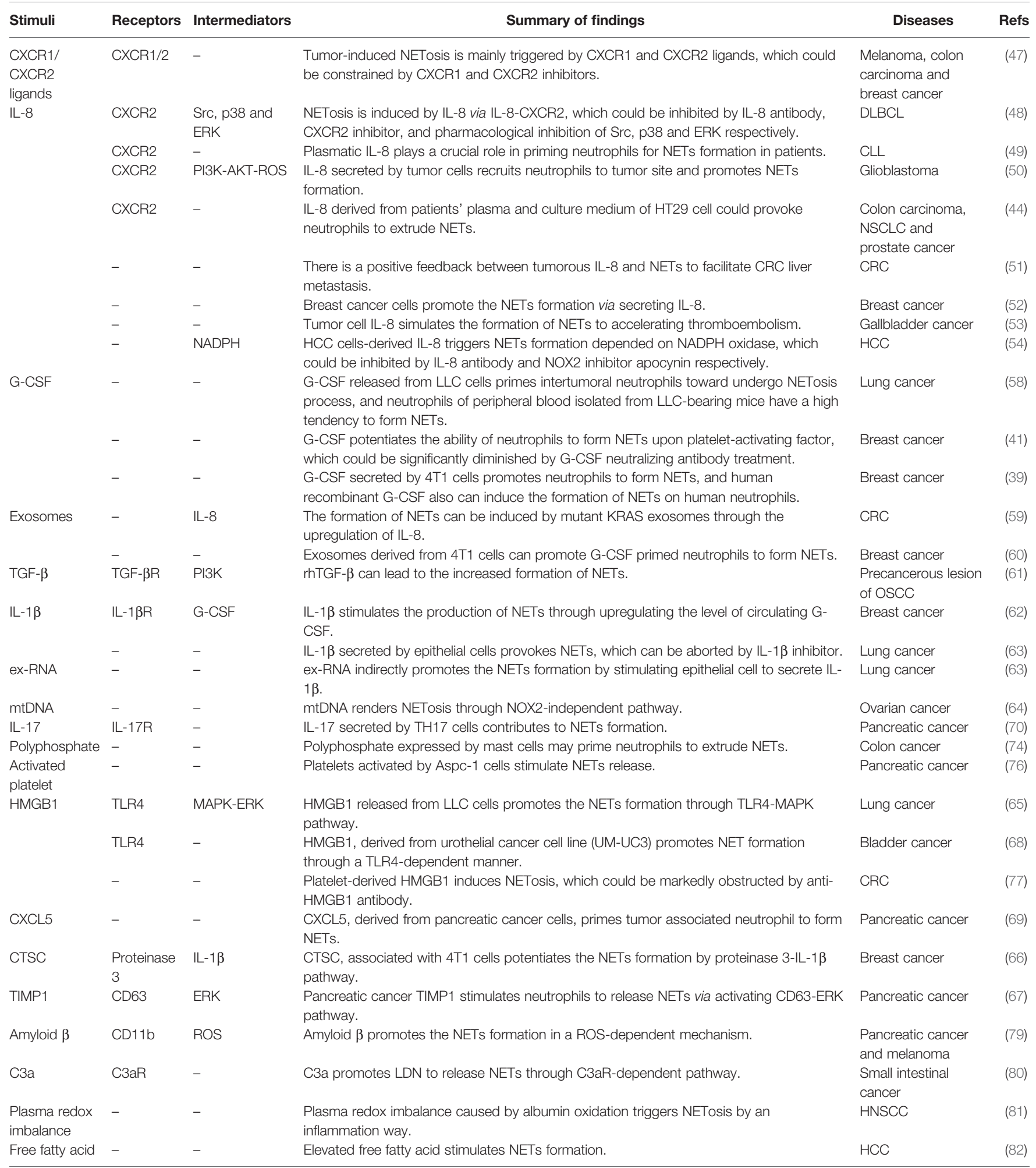

CLL, Chronic lymphocytic; CLL, Chronic lymphocytic leukemia; DLBCL, Diffuse large B-cell lymphoma; HNSCC, Head and neck squamous cell carcinoma; HCC, Hepatocellular carcinoma; NSCLC, Non-small cell lung cancer OSCC; Oral squamous cell carcinoma; HNSCC, Head and neck squamous cell carcinoma; CTSC, Cathepsin C; C3a, complement 3a; C3aR, complement 3a receptor; exRNA, extracellular RNA; HMGB1, High mobility group box-1; mtDNA, mitochondrial DNA; NADPH, Nicotinamide adenine dinucleotide phosphate; NOX2, NADPH oxidase 2; TIMP-1, Tissue inhibitor of metalloproteinases-1. No determined receptors or intermediators lead to the appearance of short bars. 
mechanism by which NETs contribute to tumor progression. Next, we will focus on the mechanism by which NETs affect tumor progression.

Given the complexity of NET components, several studies have identified NETs in their entirety to analyze their role in tumors. Researchers found that NETs promote the migration and invasion of cancer cells by activating AKT and STAT3 pathways (52). NETs also inhibit apoptosis by regulating NF$\kappa \mathrm{B}$ p65, BCL-2, and Bax expression (97). NETs have been found to be involved in the progression of diffuse large B-cell lymphoma (DLBCL) and colorectal cancer via TLR9-MAPK pathway $(48,98)$. Furthermore, a tumoral inflammatory response could be triggered by NETs through the activation of the TLR4/9-COX2 axis to fuel metastasis (38). However, considering the complexity of NETs, it is difficult to accurately clarify the role of NETs in tumors from a holistic perspective. Therefore, the roles of NET-related components have received more attention in recent years. DNA expelled by activated neutrophils serves as a cornerstone of NETs structure; and has been discovered to play a critical role in the function of NETs, because several kinds of studies demonstrate that DNase I could effectively inhibit the pro-tumor function of NETs by degrading extracellular DNA $(37,89)$. NET-DNA could act as a chemotactic factor to facilitate the migration of tumor cells to the metastatic site (99). In this process, NET-DNA could interact with extracellular $\mathrm{N}$ terminus of the coiled-coil domain containing protein 25 (CCDC25), a transmembrane protein expressed on tumor cells; $\mathrm{C}$ terminus of CCDC25 then recruits integrin-linked kinase (ILK), which subsequently stimulates $\beta$ parvin-RAC1-CDC42 cascade to facilitate tumor progression (99). NET-DNA could also promote the aggregation of platelets by binding to the receptor for advanced glycation end products (RAGE) of platelets, which contributes to the hypercoagulable state of pancreatic adenocarcinoma (100). NET-DNA could also activate the pancreatic stellate cell by upregulating the expression of p65 and pERK (101).

Apart from DNA, the complexity of NETs is determined by its protein content. Studies have confirmed that NETs include more than 20 different kinds of proteins, such as NE, MPO, calprotectin, cathelicidins, defensins, and HMGB1 (50, 102). Among these proteins, only a few studies have explored the effects and specific mechanisms of NET-related protein components on tumor progression. NET-released NE is capable of not only inducing mitochondrial dynamics, but also promoting MC38 cell proliferation by stimulating TLR4 signaling which then activates the p38-PCG1 $\alpha$ pathway (103). Tumor recurrence after treatment remains one of the main causes of cancer-associated death. Usually, metastatic cancer cells may undergo a long period of dormancy before the formation of detectable metastasis. When encountering appropriate or specific stimuli, dormant tumor cells re-enter the proliferation state and cause tumor recurrence (104). NETs have been found to mediate this process. During this process, laminin protein in the extracellular matrix (ECM) could be cleaved by NE and matrix metalloproteinase 9 (MMP9) coating on NETs; the cleaved subset of laminin could interact with integrin $\alpha 3 \beta 1$ of tumor cells, which then activates the focal adhesion kinase (FAK), MAP kinase ERK kinase (MEK), and myosin light chain 2 (MLC2) to finally awaken the dormant cancer cells (105). HMGB1 is another protein of NETs-related components under investigation. NET-HMGB1 interacts with the TLR9 of tumor cells and then stimulates TLR9 signaling which subsequently activates p38 and JNK pathways for tumor proliferation, adhesion, migration, and invasion (89). In a murine model of glioblastoma, HMGB1 contributes to IL-8 production from tumor cells through HMGB1-RAGE-ERK1/2$\mathrm{NF}-\kappa \mathrm{B}$ pathway, which may further attract circulating neutrophils to the tumor site and stimulate them to form NETs (50). Histone is also a critical protein component of NETs, and has been considered a factor that induces angiogenesis (106). Furthermore, NET-associated IL-1 $\beta$ is involved in the epithelial-to-mesenchymal transition (EMT) process of pancreatic cancer by activating EGFR-ERK pathway (107). NET-fibronectin mediates the adhesion of several human cancer cells by interacting with integrin family, such as $\alpha v \beta 5$, $\alpha v \beta 3$, and $\alpha 5 \beta 1$ (108). NET-integrin $\beta 1$ promotes the interaction between NETs and A549 cells, leading to the adhesion of lung cancer cells to liver sinusoids (109).

Part of the mechanism by which NETs promote tumor progression has been revealed in Figure 1. TLRs, integrin family, RAGE, and CCDC25 have been proven to be involved in signal transduction by direct NET binding. Furthermore, activation of NF- $\kappa B$ and MAPK plays an important role in downstream signal transduction. However, numerous questions still need to be answered. In particular, the underlying mechanism of other NET-related proteins in tumorigenesis and development remains poorly understood. Consequently, future studies may need to further explore the components of NET-related proteins, the effect and mechanism of various protein components on tumors, and the characteristics of NETs performing anti-tumor effects.

\section{ROLE OF NETS IN DIGESTIVE SYSTEM CANCERS}

The function of NETs on digestive system cancers has attracted widespread attention in recent years. Several reports have shown that NETs may perform an anti-tumor effect, but majority of existing studies suggest that NETs can exacerbate tumor-related symptoms or accentuate tumor growth, which affect the survival of patients with cancers, especially pancreatic cancer and HCC. The main functions of NETs on digestive system cancers are presented in Figure 2.

\section{Oral Cavity Cancer}

OSCC is the most common tumor of oral malignancies. Neutrophils have been found to be involved in oral cancer progression $(110,111)$, and inhibiting tumor-associated neutrophils could repress OSCC metastasis (112). Meanwhile, peripheral NLR has been demonstrated to be an independent predictor for the evaluation of overall survival (OS) for OSCC 


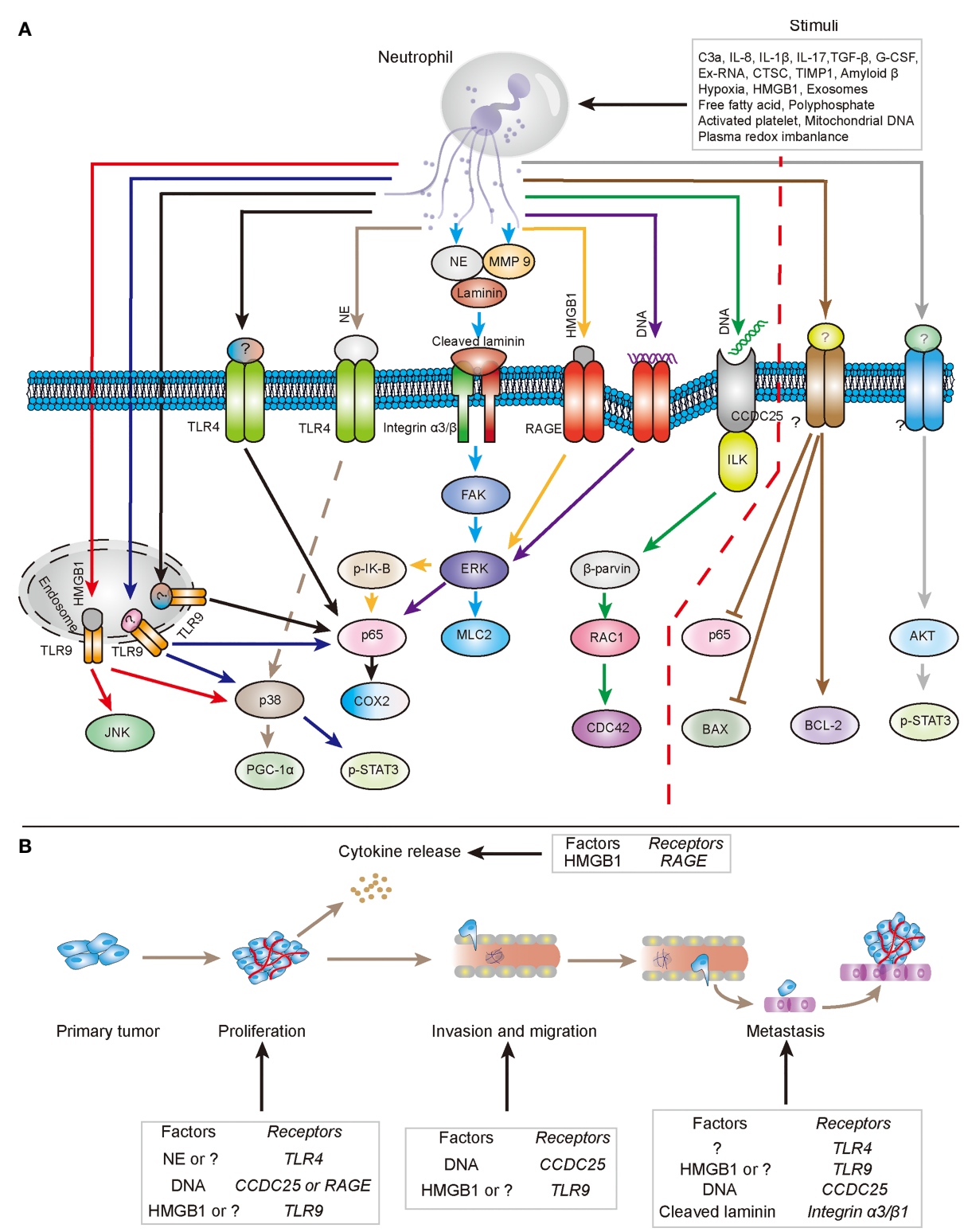

FIGURE 1 | The mechanisms by which NETs directly promote tumor progression. (A) In several types of stimuli in tumor tissues, neutrophils expel their DNA and cytoplasmic proteins to form extracellular structure to participate in tumor progression. NETs-DNA could interact with RAGE and CCDC25 respectively to be involved in the transduction of intracellular tumor-promoting signals, which facilitate tumor progression. Additionally, NETs associated protein components, such as NE, MMP9 and HMGB1, also could cooperate with respective receptor to stimulate downstream pathways to fuel the malignancy of tumors. Question marks (?) represent relevant functional components of NETs and related receptors that have not been clearly stated (especially the right side of red dotted line). (B) Components of NETs involved in the cytokine release, proliferation, invasion, migration and metastasis of tumor cells through respective relevant receptors.

patients (113). However, only a few studies have focused on the role of NETs in oral cancers.

An increase of NET formation has been discovered in the coculture system of neutrophils with OSCC cells (114). In addition, a study based on oral precancerous lesions (oral lichen planus) suggests that neutrophils stimulated by TGF- $\beta$ release more NETs, which may indicate that "N2" neutrophils may be more inclined to form NETs by upregulating PI3K after stimulation. Thus, the increased release of NETs may also be one of the mechanisms by which "N2" neutrophils promote tumor progression (61). Moreover, peripheral neutrophils in patients with Stage III/IV OSCC produce more NETs compared with healthy controls or Stage I/II patients; NETs were also found to promote the transformation of human vascular endothelial cells to a procoagulant phenotype, suggesting that elevated NETs are positively correlated with disease severity and coagulation disorders (115). However, Marzena and colleagues found that NETs in patients with inflammation and with Stage III/IV OSCC 


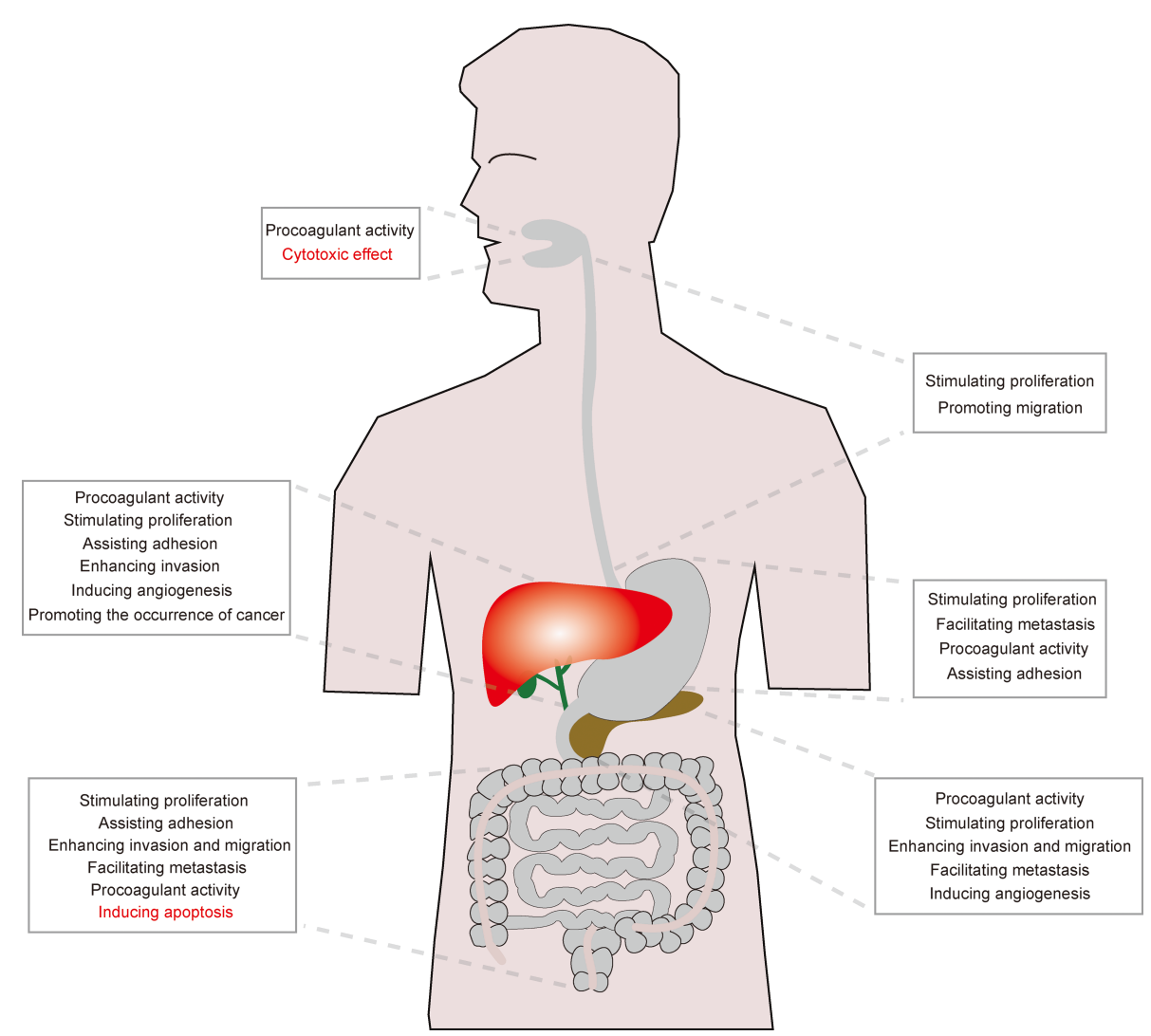

FIGURE 2 | The main effect of NETs on digestive cancers. Although the effect of NETs on OSCC and colon carcinoma remains unclear, NETs mainly play a protumor role in esophageal, gastric, pancreatic, liver and biliary cancers.

are markedly higher than those found in Stage I/II OSCC patients and healthy donors; these findings may indicate that NETs participate in the transformation of epithelial cells to malignant cells due to the stimulation of inflammation, and that NETs may play a distinct role at different stages of OSCC (116). Human neutrophil peptide-1 (HNP1) has been demonstrated to be a NET-related component (117), and found to inhibit the proliferation of OSCC cells in vitro (118), indicating that NET-related HNP1 contributes to the suppression of tumor progression.

Taken together, in response to the varying findings presented earlier, the effect and related mechanisms of OSCC on NET formation and the function of NETs on OSCC remain unclear and require further study.

\section{Esophageal Cancer}

Esophageal cancer is one of the most common tumors of the digestive system, which can be roughly divided into squamous cell carcinoma and adenocarcinoma. Regardless of the pathological type, most patients present in an advanced stage at the time of diagnosis and $80 \%$ of patients live no more than five years (119). Therefore, exploring the mechanism of esophageal cancer progression is the top priority for current research.
Circulating NETs in patients with advanced esophagogastric adenocarcinoma are significantly higher as compared with those in Stage I/II patients and healthy individuals; these findings suggest that NETs may be related to the progression of esophagogastric carcinoma (37). Another research also found that circulating cfDNA levels in patients with esophageal squamous cell carcinoma (ESCC) are significantly higher than those in control patients with benign diseases, and serum cfDNA levels are closely associated with tumor size and the host's inflammation status, such as the number of neutrophils in the peripheral blood of patients (120). Recently, a retrospective study demonstrated that tumor-infiltrating NETs are associated with dismal OS and disease-free survival (DFS) (121). NE, derived from activated neutrophils, could also promote the proliferation and progression of esophageal cancer cells by stimulating the release of cytokines, such as TGF- $\beta$, vascular endothelial growth factor (VEGF), and platelet-derived growth factor-AA (PDGFAA), which could be blocked by NE inhibitor (sivelestat) (122). However, studies on the reasons for increased NET levels in patients with esophageal cancer are still limited. A study has shown that IL-8 levels tend to be higher in patients with esophageal cancer as compared with those of healthy donors, and that IL-8 levels are closely associated with tumor size and lymph node metastasis, suggesting that increased IL-8 may be 
related to the progression of esophageal cancer (123). Considering that neutrophils are inclined to form NETs upon IL-8 stimulation (124), NET formation is believed to be correlated with IL-8 in esophageal cancer, which needs further confirmation in future studies.

Tumor progression is usually accompanied by a hypercoagulable state of blood which is closely related to the patient's prognosis. Fibrinogen and NLR score have been found to be related to tumor size, stage, invasion, and lymph node metastasis of esophageal cancer, indicating that the hypercoagulable state of blood may be closely correlated with the progression of esophageal cancer (125). However, whether NETs are involved in the formation of blood hypercoagulability in esophageal cancer remains unclear, and thus require further study.

Intriguingly, several studies aimed to explore whether sivelestat sodium, a type of NE inhibitor, could attenuate the postoperative complications of esophageal cancer patients; and these studies found that sivelestat sodium could significantly lighten postoperative hypoxia, partially reduce systemic inflammation, and maintain postoperative circulatory status, which is beneficial for the recovery of patients (126-128). However, the effect of $\mathrm{NE}$ inhibitor treatment on the subsequent progression of esophageal cancer is difficult to ascertain without the publication of follow-up date. Notably, research on NETs focuses on ESCC, and studies on esophageal adenocarcinoma are limited. Barrett's esophagus is considered a precancerous lesion of esophageal adenocarcinoma. Studies have shown that increased ROS levels of neutrophils significantly lead to esophageal mucosal injury in Barrett's esophagus, which could be retarded by the MPO inhibitor azide (129). this finding suggests that the ROS of neutrophils may be involved in the malignant transformation of Barrett's esophagus. Considering that ROS is one of the key factors in the NET formation, whether NETs are connected with the malignant transformation of Barrett's esophagus is worthy for further investigation.

\section{Gastric Cancer}

Although surgical treatment is still the main therapy for patients with gastric cancer, surgery-related complications, recurrence, and distant metastasis are considered the main cause of death. Rihito and colleagues found that low-density neutrophils (LDN), derived from abdominal cavity lavages after abdominal surgery, are prone to NET formation (130). NETs could trap the human gastric cancer cell lines (i.e., MKN45, OCUM-1, NUGC-4) in vitro to promote their proliferation (130), facilitate a more aggressive mesenchymal phenotype of AGS cells (131), and strongly augment the metastasis of MKN45 cells on peritoneum, which could be inhibited by IP administration with DNase I (132); these findings suggest that NETs play an important role in peritoneal metastasis of gastric cancer. In view of NETs that can trap tumor cells and promote their survival, whether NETs in the circulatory system can trap gastric cancer cells and assist them in the metastasis to a distant organ are yet to be determined.

Circulating neutrophils in gastric cancer patients are prone to form NETs spontaneously compared with those in healthy donors (133), NET levels are significantly associated with neutrophil count and NLR in the peripheral blood (134). Roni and colleagues found that circulating NETs are significantly elevated in patients with advanced gastric tumors, compared with those in local cancer patients and healthy controls, and closely associated with disease stage (37). Interestingly, NETs could serve as a prognostic factor, which may be more sensitive to predicting the outcome of patients compared to carcinoembryonic antigen (CEA) and carbohydrate antigen 199 (CA199) of serum (134). Moreover, increased LDN production has been found to be positively correlated with operative time and intraoperative blood loss, and LDN is prone to form NETs which served as a "carrier" and an "armor" for transporting and protecting gastric cancer cells to facilitate distant metastasis (135). However, surgery stress is not a necessary condition for NETosis; tumor itself can also induce NET formation (37). In addition to directly contacting with tumor cells, NETs may be involved in coagulation abnormality of patients with gastric cancer. The NET levels of gastric cancer patients have been found to be significantly correlated with thrombin-antithrombin complex levels and D-dimers, indicating that NETs may play a procoagulant role in the progression of gastric cancer (133). Preoperative blood coagulation status is closely related to lymph node metastasis and disease stage of patients suffering from gastric cancers; this status is also considered to be one of the main causes of death (136). Therefore, targeting NETs therapy may suppress metastasis and recurrence and improve prognosis in gastric cancer patients.

Regarding the relationship between NETs and gastric cancer, existing research shows that NETs contribute to the hypercoagulable state of the circulating system and may promote metastasis of the abdominal cavity and distant organs. However, the direct effect of NETs on gastric cancer cells and tumor angiogenesis and the influence of NETs on other immune cells in the TME remain largely unknown. The related mechanisms of NETs promoting gastric cancer metastasis still require more detailed analysis. A recent study used neutrophils loaded with Abraxane (nanoparticle albumin bound paclitaxel) to serve as a Trojan horse; when they reach the radiated tumor site, the Abraxane could be released into tumor via NETosis and exert tumor killing function (137). The above research reveals that researchers should not only explore the role of NETs in tumorigenesis and development but also the utilization of NETs for cancer treatment.

\section{Colorectal Cancer}

Liver metastasis and postoperative recurrence are the main factors for the poor prognosis of colon cancer (138). As mentioned earlier, surgery stress could induce neutrophils to form NETs, which are correlated positively with operative time and intraoperative blood loss (135). Similar to this finding, a study showed that the levels of postoperative circulating NETs are negatively related to the DFS of patients undergoing attempted curative liver resection for metastatic CRC; in addition, abnormal NETs in the tumor are closely correlated with intratumor hypoxia in a murine model (89). Regardless of 
stimuli, neutrophils from patients with CRC also tend to undergo NETosis in vitro compared with those of healthy donors (90). Moreover, poor outcomes of patients are significantly correlated with increased preoperative NET production (103). Those studies revealed that several factors, such as exosome with Kras mutation (59), poly-phosphate (74), and platelet (77), may induce abnormal NET production in the serum of CRC patients, aside from surgery stimulation.

What role does NETs play in the tumorigenesis and development of CRC? Several reports have shown that NETs are involved in CRC progression by promoting tumor cell proliferation, invasion, and adhesion (37, 51, 89, 99). NETs activate the TLR-9 pathway via NET-related HMGB1 to promote CRC cell proliferation, adhesion, migration and metastasis, which can be inhibited by DNase I administration (89). Clinical research revealed that NETs are abundant in the liver metastasis of patients with colon cancer, and NET-DNA functions as a pro-tumor factor that promotes tumor cell proliferation, adhesion, migration and metastasis by interacting with the extracellular $\mathrm{N}$ terminus of CCDC25 protein in a preclinical murine model (99). NE, one component of NETs, is found to be correlated with CRC progression, and $\mathrm{NE}$ concentration is not only significantly higher in the tumor tissues than the normal tissues; but is also higher in the serum of CRC patients compared with those of healthy controls (139). $\mathrm{NE}$ has also been found to be a pro-tumor factor and to stimulate the MC38 cell proliferation (103). Furthermore, NETs contribute to the coagulation dysfunction of CRC patients by impairing endothelial cells and activating platelets to shortening coagulation time, and increasing thrombin-antithrombin (TAT) complexes and fibrin fibrils (77). MMP-9, a component of NETs, has been found to promote liver metastasis of colon cancer in an ischemia-reperfusion injury model of the liver, which can be prevented by doxycycline, a broad-spectrum MMP inhibitor (140), further indicating that NETs may be involved in the metastatic process of CRC. On the other hand, NETs are not only capable of modifying the metabolic programming of cancer cells to reduce tumor cell apoptosis and enhance tumor cell growth in vitro but can also promote the development of subcutaneous tumor implants and hepatic metastases in mice (103). Although most studies have demonstrated that NETs mainly promote CRC progression, NETs have also been proven to inhibit the proliferation and induce apoptosis of Coca 2 cells in vitro (85). Although these reports generally indicate that NETs could be involved in CRC progression, such as promoting tumor growth, leading the hypercoagulation state of the circulatory system, and mediating the liver metastasis, the mechanisms by which NETs promote CRC progression are still unclear, and must be confirmed in future research. In the view of NET-associated anti-tumor function, NETs may perform different functions on CRC progression under certain conditions, which must also be further explored.

In addition to studies on the relationship between NETs and CRC, several studies focused on diseases that are closely related to CRC, such as inflammatory bowel disease and intestinal polyps. Chronic inflammation has been proven to promote the malignant transformation of epithelial cells and is closely related to the occurrence of tumors. Crohn's disease and ulcerative colitis (UC) are the most common inflammatory bowel diseases (IBD), which are closely related to the occurrence of colitis-associated colorectal cancer (141). Considering that IBD is regarded as a risk factor for CRC $(142,143)$, the role of NETs in the progression of IBD needs to be explored. Studies have shown an abnormal increase of NET-related proteins in fecal samples from IBD patients (144) and in UC colon tissue (145). NETassociated proteins have also been found to be over-expressed in the inflamed colon of UC patients compared with healthy controls. Colitis could be effectively attenuated by NETs inhibition in a dextran sodium sulfate induced mouse model (146). Fecal calprotectin, one component of NET-associated proteins, has been suggested to be a reliable marker for the detection of IBD activity (147). These studies confirm that NETs play a key role in the occurrence and progression of IBD, which is further demonstrated in pediatric IBD (148). However, the specific mechanisms of NETs that promote IBD progression, and whether NETs could accelerate the malignant transformation of IBD require further study. Intestinal polyps and colorectal adenomas, as precancerous lesions of colorectal cancer, have been studied extensively. A study has shown that NETs are involved in the progression of CRC and adenomas with highgrade dysplasia (74). Furthermore, calprotectin has been demonstrated to participate in the progression of colorectal polyps (149). MMP9 has also been found to activate latent TGF- $\beta$, which then further suppresses the T-cell activity to promote the occurrence and development of CRC in the model of Apc $2^{\mathrm{fl} / \mathrm{fl}-\mathrm{Cd} 2 \mathrm{CreERT}}$ mice (150). Prevention is considered one of the best ways to treat cancer. Therefore, further research on the roles of NETs in IBD, polyps, and adenomas are largely helpful to the prevention and treatment of CRC.

In general, NETs have been widely proven to play a critical role in CRC progression, but the specific underlying mechanism remains largely unknown. Meanwhile, NETs have been found to contribute to the development of IBD and polyps which may facilitate tumor formation. Thus, targeting NETs could contribute to the prevention and treatment of CRC. A recent study showed that targeting NET-associated carcinoembryonic Ag cell adhesion molecule 1 (CEACAM1) could inhibit the adhesion, migration and metastasis of colon cancer cells, which indicates that CEACAM 1 may serve as a potential therapeutic target to retard CRC progression (151).

\section{Liver and Biliary Cancer}

Liver cancer is the seventh most frequently diagnosed cancer and the second most common cause of cancer-related death all over the world (14). Among liver cancers, hepatocellular carcinoma (HCC) accounts for $70 \%$ to $85 \%$ of patients with confirmed liver cancer worldwide. Several factors have been found to be involved in the occurrence of HCC, such as hepatitis B or C virus, metabolic disorders, nonalcoholic steatohepatitis, or alcohol intoxication (152). NETs have been found to contribute to the progression of various chronic liver diseases, such as 
alcohol-associated liver disease, nonalcoholic fatty liver disease and portal hypertension (153), which are closely related to HCC occurrence, indicating that NETs may play a role in HCC development.

Studies have shown that NETs levels are higher in patients with nonalcoholic steatohepatitis than those in healthy individuals. Analyses on potential mechanism have indicated that early infiltration of neutrophils and the increase of NETs promote the monocyte infiltration and inflammatory cytokines production (82), and induce the differentiation of naive $\mathrm{T}$ cell into Treg by activating TLR4 signaling (154), which then result in malignant transformation of epithelial cells and development of liver cancer. Furthermore, serum NETs in HCC patients are also significantly higher than those in healthy donors (155). Increased NETs have been demonstrated to be closely related to the formation of portal vein thrombosis in patients with HCC and positively correlated with liver disease severity (156). However, a single center, prospective tissue-based study found no correlation between NETs and the venous thrombosis of patients with liver cancer (157). Considering that numerous factors affect coagulation function and that the abnormal liver function itself is also closely related to coagulation abnormality, the relationship between NETs and blood hypercoagulability in patients with liver cancer may require further large-scale clinical studies and more experimental data to determine the effects of NETs on coagulation of live cancer patients. Aside from the capability of NETs to promote malignant transformation of epithelial cells and its involvement in the hypercoagulation status of patients, NETs have also been found to directly stimulate Hepa1-6 and Huh 7 cell proliferation by enhancing mitochondrial function and biogenesis in vitro, similar to findings in CRC research (103); NETs could also capture circulating cancer cells, which leads to hepatic malignancy metastasis, depending on the activated platelet (158). Moreover, neutrophils derived from HCC patients, especially advanced HCC, are inclined to release NETs even without any stimulation, and plasma of patients or culture medium of HCC cells could stimulate the release of NETs in neutrophils of healthy donors (38). Another study showed that NETs have no effect on the proliferation of HCC cells in vitro but can promote tumor growth in vivo by enhancing adhesion, invasion, and angiogenesis of HCC, suggesting that tumor proliferation led by NETs may partly or completely require the participation of other factors in HCC (38). Additionally, cathepsin G associated with NETs improves the invasive ability of HCC by regulating the expression of E-cadherin in vitro and in vivo (54). These results indicate that NETs are rich in patients with nonalcoholic steatohepatitis and HCC, which play a critical role in the occurrence and development of liver cancer by promoting angiogenesis, adhesion and invasion of the tumor. However, whether NETs promote the hypercoagulable state of the blood is still unclear. Existing studies on the roles of NETs in liver cancer, such as whether NETs are related to liver cancer metastasis and the metabolism of vitamin $K$, are still not sufficiently comprehensive. Recent studies found that NETs may play a vital role in the formation and development of portal hypertension (PHTN) $(159,160)$. These findings indicate that whether NETs are involved in the formation of PHTN and liver cancer-associated ascites also needs to be studied. Therefore, indepth research is still necessary to determine the effect of NETs on liver cancer.

Hepatitis virus infection is widely known as a common cause of liver cancer. Related studies revealed that the peripheral NLR of patients with chronic hepatitis B is positively correlated with disease severity and mortality (161). A recent study showed that HBV infection can inhibit the release of NETs from neutrophils by regulating the levels of cellular ROS and autophagy to maintain chronic liver inflammation (162). The abovementioned studies suggest that neutrophils may affect the outcome of viral hepatitis, but their specific role is still unclear, and whether NETs play a role in the final malignant transformation of viral hepatitis needs to be further explored.

Cholangiocarcinoma and gallbladder cancer also are common malignant tumors of the biliary system. Most patients experience biliary obstruction, worsening of progressive jaundice, and poor prognosis. Research focusing on the role of NETs in those two tumors is limited. Studies indicate that neutrophil infiltration in the tumor is negatively related to the prognosis of patients with cholangiocarcinoma $(163,164)$. Infiltrative intertumoral neutrophils recruited by CXCL5 could promote tumor metastasis and the recurrence of intrahepatic cholangiocarcinoma (165). Additionally, the level of NETs in the peripheral blood of patients with extrahepatic cholangiocarcinoma is significantly higher than that in healthy people, and NETs levels are positively correlated with the occurrence of thrombosis in patients (166). A recent clinical study showed that the peripheral NLR (NLR $\geq 5)$ could be regarded as an independent prognostic factor for gallbladder cancer, suggesting poor prognosis (167). Meanwhile, NETs accelerate the formation of thromboembolism in gallbladder cancer (53). Related studies suggest that neutrophils may be involved in the progression of cholangiocarcinoma and gallbladder cancer, but the specific mechanism is still unknown. Whether NETs play a major role in this process requires further study.

\section{Pancreatic Cancer}

Most patients with pancreatic cancer are at an advanced stage at the time of diagnosis, and metastasis and thrombosis are the main factors affecting the prognosis of patients $(168,169)$. A retrospective cohort study showed that approximately $40 \%$ of patients with advanced pancreatic cancer are diagnosed with venous thromboembolism (VTE) after the start of palliative chemotherapy (170). Moreover, VTE may be negatively related to the chemotherapeutic effect, progression-free survival and OS of patients with advanced pancreatic cancer (171). Abnormally increased NETs are observed in pancreatic cancer patients and the models of murine orthotopic pancreatic cancer $(95,172)$; these NETs can be regarded as an independent prognostic factor for evaluating the outcomes of patients with PDAC, which are negatively correlated with the OS and recurrence-free survival of patients (40). Existing studies showed that increased levels of NETs in pancreatic cancer are partly due to the direct effect of tumor cells, cancer-associated fibroblasts, or activated platelet $(67,76,79,106)$. 
NETs have been found to be closely related to blood hypercoagulability in patients with pancreatic cancer (173); and in nude mice with human pancreatic cancer cells (174) by several ways, such as converting endothelial cells toward a procoagulant phenotype (95) and stimulating platelet aggregation $(76,106)$. Furthermore, NETs have been found to cooperate with procoagulant microparticles released by tumor cells to promote the formation of deep vein thrombosis in murine models of PDAC (175). NET formation could be restricted by inhibiting autophagy with chloroquine or genetic ablation of RAGE, indicating that NETosis in pancreatic cancer may be mainly dependent on autophagy or RAGE expression of neutrophil (172). In addition, a randomized controlled clinical study found that chemotherapy with hydroxychloroquine significantly reduces peri-operative VTE rate (100). These results suggest that NETs could promote the formation of venous thrombosis of pancreatic cancer patients through several ways, which then facilitate disease progression. Therefore, treatment of NETs may reduce the mortality of patients, and improve the quality of life and prognosis of patients with pancreatic cancer.

NETs have been found to significantly promote tumor angiogenesis and potentiate the migration and invasion abilities of AsPC-1 PANC-1 and MMIAPaCa-2 cell in vitro $(106,176)$. NETassociated IL-1 $\beta$ was recently found responsible for the EMT process of pancreatic cancer via EGFR-ERK pathway (107). In addition to the direct effect, NETs can facilitate tumor progression by affecting the functions of other cells. NETs could also promote and enable tumor proliferation by stimulating pancreatic stellate cells that form dense fibrous stroma (101). NETs also enhance the migration of hepatic stellate cells, which then promote liver micrometastasis of PDAC (177).

The role of NETs in pancreatic cancer is relatively clear, which is mainly to promote tumor progression. However, the regulation of NETs on tumor cells, angiogenesis, and metastasis remains unclear. Moreover, NETs have been found to impair the sensitivity of PDAC to the treatment of immune checkpoint blockade (PD-1, CTLA4) (72). Thus, whether the combination between targeting NETs and other treatment is beneficial to the prognosis of pancreatic cancer will need to be further explored.

\section{DISCUSSION}

\section{Insights About This Double-Edged Sword}

Like a double-edged sword, NETs have been proven to perform an anti-tumor or pro-tumor function on tumor progression. The determinant of the difference of NETs-associated function on tumor remains unknown. As shown in Figure 1, the complexity of inducing factors and components may determine the characteristics of the double-edged sword of NETs in cancer. Therefore, in-depth study on the inducing factors and components of NETs must be conducted, which may gradually reveal the role of NETs in cancer.

Neutrophils are widely known as a type of multi-functional and complex cells; that are not only involved in the innate immune response, but also in modulating the adaptive immune response by directly or indirectly affecting the dendritic cells and lymphocytes (178). Therefore, neutrophils have been considered as a bridge between the innate and adaptive immunity. To determine the role of NETs in cancer, their effect on the innate and adaptive immunity, especially in the tumor immune microenvironment, requires research. NETs have also been found to impair the anti-tumor function of $\mathrm{CD}^{+} \mathrm{T}$ lymphocytes and NK cells (47), further indicating that exploring the role of NETs in immune response is crucial for cancer treatment. In addition, whether NETs can affect the function of macrophages and dendritic cells (DCs) needs to be determined. NETs could dampen the LPS-induced maturation of DCs, whereas DCs also could promote the degradation of NETs, suggesting that an interaction transpires between NETs and DCs (179). Furthermore, a study has shown the roles of NETs in macrophages and DCs may be partly dependent on the duration of NETs. When exposed to NETs, macrophages and DCs have been activated at an early time (30 min) but induced to apoptosis at a later time (6 and 24 hours) (180). These results indicate that NETs may impair the function of both antigen-presenting cells (APCs) and can further delay their own degradation process. However, studies on the relationship and interaction between NETs and APCs in the context of tumor are limited and may need further exploration.

Notably, neutrophils show distinct phenotypic and functional properties at different times of the day, in a process named as aging (181). Approximately 40\% of neutrophils in the blood of healthy individuals express CD177 protein (182). Furthermore, $\mathrm{CD}_{177^{+}}$neutrophils have been found to be prone to NETs formation and to produce higher levels of IL-22 and TGF- $\beta$ compared with CD177- neutrophils (183). In addition, several subsets of neutrophils have been found in the context of cancer, such as tumor-associated neutrophils (TAN) and myeloidderived suppressor cells (MDSC) $(178,184)$. The idea that neutrophils are a homogeneous population can therefore be considered unreliable. Consequently, several questions still exist, such as whether each subset of neutrophils can produce NETs upon stimuli, and whether the produced NETs are different from each other. These questions need to be addressed in future research to clarify the role of NETs in cancer.

Overall, although further research is necessary, NETs have been confirmed to play a role in tumors, in terms of both antiand pro-tumor functions. Most of the existing studies suggest that NETs mainly play a role in promoting tumors, and targeting NETs may serve as a hopeful treatment for tumors. Therefore, exploring ways to target NETs may be beneficial for cancer treatment.

\section{Strategies to Suppress NETs Formation}

Based on the theory that NETs mainly fuel tumor progression (Figures 1 and 2), the role of NETs in tumors can be reversed by interfering with NET formation from the perspective of the mechanism of NET formation or from the tumor-related factors that induce NETosis. Theoretically, five strategies can be adopted to inhibit NET formation.

First, the abnormally increased NETs in the tumor area can be reduced by inhibiting the infiltration of neutrophils into the 
tumor site. Second, the related factors of tumor that induce NET formation (Table 1) can be targeted to reduce the abnormal levels of NETs in the tumor site and circulatory system. Third, NET formation can be interfered by targeting neutrophils themselves, such as inhibiting autophagy with chloroquine (172) or disturbing the function of PAD4 protein by GSK484 (92) or BMS-P5, a novel PAD4 inhibitor (185). Furthermore, the pro-tumor effects of NETs can be suppressed by blocking the signaling pathways (Figure 1) by which NETs act as a pro-tumor stimulation. Lastly, accelerating the degradation of NETs can help in attenuating the tumor-promoting effects of NETs, such as DNase I administration $(37,48,101)$. Recently, liver metastasis of CRC can be inhibited by gene therapy of adeno-associated virus vector which specifically expresses DNase I in the liver (186), further suggesting that treatment with DNase I is a potential cancer treatment.

Served as an arm of neutrophils, NETs notably play an important role in the infection. Although IP administration with DNase I or PAD4 inhibitor has been demonstrated to be safe in several murine models of cancer, whether systemic administration will increase the infection rate remains unclear. Considering the safety of targeting NETs in clinical applications, the clinical application of existing NETs inhibitors may first be used for the adjuvant treatment of tumors. Dornase alfa, a recombinant desoxyribonuclease, has been approved by the US Food and Drug Administration (FDA) for the treatment of cystic fibrosis, suggesting that administration with DNase I is tolerable and safe for patients. Additionally, several clinical trials are exploring the effectiveness of DNase I nebulized inhalation to reduce lung symptoms in patients with respiratory distress syndrome (NCT03368092) or COVID-19 (NCT04541979, NCT04402970, NCT04387786, NCT04359654). Furthermore, Danirixin (GSK1325756), a selective CXCR2 antagonist, has been found to alleviate the symptoms of patients with chronic obstructive pulmonary disease (COPD) by inhibiting the

\section{REFERENCES}

1. Nathan C. Neutrophils and Immunity: Challenges and Opportunities. Nat Rev Immunol (2006) 6:173-82. doi: 10.1038/nri1785

2. Coffelt SB, Wellenstein MD, de Visser KE. Neutrophils in Cancer: Neutral No More. Nat Rev Cancer (2016) 16:431-46. doi: 10.1038/nrc.2016.52

3. Nemeth T, Sperandio M, Mocsai A. Neutrophils as Emerging Therapeutic Targets. Nat Rev Drug Discov (2020) 19:253-75. doi: 10.1038/s41573-0190054-z

4. Brinkmann V, Reichard U, Goosmann C, Fauler B, Uhlemann Y, Weiss DS, et al. Neutrophil Extracellular Traps Kill Bacteria. Science (2004) 303:15325. doi: 10.1126/science.1092385

5. Urban CF, Reichard U, Brinkmann V, Zychlinsky A. Neutrophil Extracellular Traps Capture and Kill Candida Albicans Yeast and Hyphal Forms. Cell Microbiol (2006) 8:668-76. doi: 10.1111/j.1462-5822. 2005.00659.x

6. Abi Abdallah DS, Lin C, Ball CJ, King MR, Duhamel GE, Denkers EY. Toxoplasma Gondii Triggers Release of Human and Mouse Neutrophil Extracellular Traps. Infect Immun (2012) 80:768-77. doi: 10.1128/ IAI.05730-11

7. Saitoh T, Komano J, Saitoh Y, Misawa T, Takahama M, Kozaki T, et al. Neutrophil Extracellular Traps Mediate a Host Defense Response to Human formation of NETs (NCT03250689) (187). Moreover, sivelestat, a NE inhibitor, which has been used in operative patients to attenuate post-operative complications (NCT01170845), is also promising for cancer treatment.

In summary, exploring the specific role of NETs in tumors is top priority. Meanwhile, it is necessary to study the effectiveness of existing targeting NETs treatment in cancer. Finally, the role of NETs in predicting tumor occurrence and prognosis cannot be ignored.

\section{AUTHOR CONTRIBUTIONS}

YC and LH collected related papers and wrote the initial manuscript. XQ contributed to the figure and table. GW and JZ designed the study and revised the manuscript. All authors contributed to the article and approved the submitted version.

\section{FUNDING}

This work was supported by the National Natural Science Foundation of China (81871869 and 82072814); Jiangsu Province Social Development Key Projects (BE2020641 and BE2020640); Key Research Development project of Xuzhou (KC19082); the Natural Science Key Project of Jiangsu Provincial Education Department (19KJA470001); Youth Technology Innovation Team of Xuzhou Medical University (TD202003); Jiangsu Provincial Key Medical Discipline, The Project of Invigorating Health Care through Science, Technology and Education (ZDXKA2016014 and CXTDA2017034); Postgraduate Research \& Practice Innovation Program of Jiangsu Province (KYCX20_2458) and the Qing Lan Project of Jiangsu Province.
Immunodeficiency Virus-1. Cell Host Microbe (2012) 12:109-16. doi: 10.1016/j.chom.2012.05.015

8. Zuo Y, Yalavarthi S, Shi H, Gockman K, Zuo M, Madison JA, et al. Neutrophil Extracellular Traps in COVID-19. JCI Insight (2020) 5: e138999. doi: 10.1101/2020.04.09.20059626

9. Middleton EA, He XY, Denorme F, Campbell RA, Ng D, Salvatore SP, et al. Neutrophil Extracellular Traps Contribute to Immunothrombosis in COVID-19 Acute Respiratory Distress Syndrome. Blood (2020) 136:116979. doi: 10.1182/blood.2020007008

10. Ng H, Havervall S, Rosell A, Aguilera K, Parv K, von Meijenfeldt FA, et al Circulating Markers of Neutrophil Extracellular Traps Are of Prognostic Value in Patients With COVID-19. Arterioscler Thromb Vasc Biol (2021) 41:988-94. doi: 10.1161/ATVBAHA.120.315267

11. Barnes BJ, Adrover JM, Baxter-Stoltzfus A, Borczuk A, Cools-Lartigue J, Crawford JM, et al. Targeting Potential Drivers of COVID-19: Neutrophil Extracellular Traps. J Exp Med (2020) 217:e20200652. doi: 10.1084/ jem.20200652

12. Garley M, Jablonska E, Dabrowska D. NETs in Cancer. Tumour Biol (2016) 37:14355-61. doi: 10.1007/s13277-016-5328-Z

13. Yuan X, Yuan Y, He Z, Li D, Zeng B, Ni Q, et al. The Regulatory Functions of Circular RNAs in Digestive System Cancers. Cancers (Basel) (2020) 12:770. doi: $10.3390 /$ cancers 12030770 
14. Bray F, Ferlay J, Soerjomataram I, Siegel RL, Torre LA, Jemal A. Global Cancer Statistics 2018: GLOBOCAN Estimates of Incidence and Mortality Worldwide for 36 Cancers in 185 Countries. CA Cancer J Clin (2018) 68:394-424. doi: 10.3322/caac.21492

15. Hedrick CC, Malanchi I. Neutrophils in Cancer: Heterogeneous and Multifaceted. Nat Rev Immunol (2021). doi: 10.1038/s41577-021-00571-6

16. Phulari RGS, Rathore RS, Shah AK, Agnani SS. Neutrophil: Lymphocyte Ratio and Oral Squamous Cell Carcinoma: A Preliminary Study. J Oral Maxillofac Pathol (2019) 23:78-81. doi: 10.4103/jomfp.JOMFP_160_17

17. Chen MF, Chen PT, Kuan FC, Chen WC. The Predictive Value of Pretreatment Neutrophil-To-Lymphocyte Ratio in Esophageal Squamous Cell Carcinoma. Ann Surg Oncol (2019) 26:190-9. doi: 10.1245/s10434-0186944-1

18. Jiang C, Hu WM, Liao FX, Yang Q, Chen P, Rong YM, et al. Elevated Preoperative Neutrophil-to-Lymphocyte Ratio Is Associated With Poor Prognosis in Gastrointestinal Stromal Tumor Patients. Onco Targets Ther (2016) 9:877-83. doi: 10.2147/OTT.S90569

19. Chu ZQ, Zhang KC, Chen L. Neutrophil Extracellular Traps in Gastrointestinal Cancer. World J Gastroenterol (2021) 27:5474-87. doi: 10.3748/wjg.v27.i33.5474

20. Sorvillo N, Cherpokova D, Martinod K, Wagner DD. Extracellular DNA NET-Works With Dire Consequences for Health. Circ Res (2019) 125:47088. doi: 10.1161/circresaha.119.314581

21. Zychlinsky A, Brinkmann V, Weinrauch Y, Wahn V, Schulze I, Hurwitz R, et al. Novel Cell Death Program Leads to Neutrophil Extracellular Traps. J Cell Biol (2007) 176:231-41. doi: 10.1083/jcb.200606027

22. Pilsczek FH, Salina D, Poon KKH, Fahey C, Yipp BG, Sibley CD, et al. A Novel Mechanism of Rapid Nuclear Neutrophil Extracellular Trap Formation in Response Tostaphylococcus Aureus. J Immunol (2010) 185:7413-25. doi: 10.4049/jimmunol.1000675

23. Hakkim A, Fuchs TA, Martinez NE, Hess S, Prinz H, Zychlinsky A, et al. Activation of the Raf-MEK-ERK Pathway Is Required for Neutrophil Extracellular Trap Formation. Nat Chem Biol (2011) 7:75-7. doi: 10.1038/ nchembio. 496

24. Papayannopoulos V, Metzler KD, Hakkim A, Zychlinsky. Neutrophil elastase A. And Myeloperoxidase Regulate the Formation of Neutrophil Extracellular Traps. J Cell Biol (2010) 191:677-91. doi: 10.1083/ jcb. 201006052

25. Lood C, Blanco LP, Purmalek MM, Carmona-Rivera C, De Ravin SS, Smith CK, et al. Neutrophil Extracellular Traps Enriched in Oxidized Mitochondrial DNA Are Interferogenic and Contribute to Lupus-Like Disease. Nat Med (2016) 22:146-53. doi: 10.1038/nm.4027

26. Douda DN, Khan MA, Grasemann H, Palaniyar N. SK3 Channel and Mitochondrial ROS Mediate NADPH Oxidase-Independent NETosis Induced by Calcium Influx. Proc Natl Acad Sci (2015) 112:2817-22. doi: 10.1073/pnas.1414055112

27. Hosseinzadeh A, Thompson PR, Segal BH, Urban CF. Nicotine Induces Neutrophil Extracellular Traps. J Leukoc Biol (2016) 100:1105-12. doi: 10.1189/jlb.3AB0815-379RR

28. Mor-Vaknin N, Saha A, Legendre M, Carmona-Rivera C, Amin MA, Rabquer BJ, et al. DEK-Targeting DNA Aptamers as Therapeutics for Inflammatory Arthritis. Nat Commun (2017) 8:14252. doi: 10.1038/ ncomms 14252

29. Rohrbach AS, Slade DJ, Thompson PR, Mowen KA. Activation of PAD4 in NET Formation. Front Immunol (2012) 3:360. doi: 10.3389/ fimmu.2012.00360

30. Wang Y, Wysocka J, Sayegh J, Lee YH, Perlin JR, Leonelli L, et al. Human PAD4 Regulates Histone Arginine Methylation Levels via Demethylimination. Science (2004) 306:279-83. doi: 10.1126/science. 1101400

31. Kolaczkowska E, Jenne CN, Surewaard BG, Thanabalasuriar A, Lee WY, Sanz MJ, et al. Molecular Mechanisms of NET Formation and Degradation Revealed by Intravital Imaging in the Liver Vasculature. Nat Commun (2015) 6:6673. doi: 10.1038/ncomms7673

32. Cools-Lartigue J, Spicer J, McDonald B, Gowing S, Chow S, Giannias B, et al. Neutrophil Extracellular Traps Sequester Circulating Tumor Cells and Promote Metastasis. J Clin Invest (2013) 123:3446-58. doi: 10.1172/JCI67484

33. Bawadekar M, Shim D, Johnson CJ, Warner TF, Rebernick R, Damgaard D, et al. Peptidylarginine Deiminase 2 Is Required for Tumor Necrosis Factor
Alpha-Induced Citrullination and Arthritis, But Not Neutrophil Extracellular Trap Formation. J Autoimmun (2017) 80:39-47. doi: 10.1016/j.jaut.2017.01.006

34. Branzk N, Lubojemska A, Hardison SE, Wang Q, Gutierrez MG, Brown GD, et al. Neutrophils Sense Microbe Size and Selectively Release Neutrophil Extracellular Traps in Response to Large Pathogens. Nat Immunol (2014) 15:1017-25. doi: 10.1038/ni.2987

35. Hakkim A, Furnrohr BG, Amann K, Laube B, Abed UA, Brinkmann V, et al. Impairment of Neutrophil Extracellular Trap Degradation Is Associated With Lupus Nephritis. Proc Natl Acad Sci USA (2010) 107:9813-8. doi: 10.1073/pnas.0909927107

36. Farrera C, Fadeel B. Macrophage Clearance of Neutrophil Extracellular Traps Is a Silent Process. J Immunol (2013) 191:2647-56. doi: 10.4049/ jimmunol.1300436

37. Rayes RF, Mouhanna JG, Nicolau I, Bourdeau F, Giannias B, Rousseau S, et al. Primary Tumors Induce Neutrophil Extracellular Traps With Targetable Metastasis Promoting Effects. JCI Insight (2019) 5:e128008. doi: $10.1172 /$ jci.insight. 128008

38. Yang LY, Luo Q, Lu L, Zhu WW, Sun HT, Wei R, et al. Increased Neutrophil Extracellular Traps Promote Metastasis Potential of Hepatocellular Carcinoma via Provoking Tumorous Inflammatory Response. J Hematol Oncol (2020) 13:3. doi: 10.1186/s13045-019-0836-0

39. Park J, Wysocki RW, Amoozgar Z, Maiorino L, Fein MR, Jorns J, et al. Cancer Cells Induce Metastasis-Supporting Neutrophil Extracellular DNA Traps. Sci Transl Med (2016) 8:361ra138. doi: 10.1126/scitranslmed.aag1711

40. Jin W, Xu HX, Zhang SR, Li H, Wang WQ, Gao HL, et al. Tumor-Infiltrating NETs Predict Postsurgical Survival in Patients With Pancreatic Ductal Adenocarcinoma. Ann Surg Oncol (2019) 26:635-43. doi: 10.1245/s10434018-6941-4

41. Demers M, Krause DS, Schatzberg D, Martinod K, Voorhees JR, Fuchs TA, et al. Cancers Predispose Neutrophils to Release Extracellular DNA Traps That Contribute to Cancer-Associated Thrombosis. Proc Natl Acad Sci USA (2012) 109:13076-81. doi: 10.1073/pnas.1200419109

42. Berger-Achituv S, Brinkmann V, Abed UA, Kuhn LI, Ben-Ezra J, Elhasid R, et al. A Proposed Role for Neutrophil Extracellular Traps in Cancer Immunoediting. Front Immunol (2013) 4:48. doi: 10.3389/fimmu.2013.00048

43. Cedervall J, Zhang Y, Huang H, Zhang L, Femel J, Dimberg A, et al. Neutrophil Extracellular Traps Accumulate in Peripheral Blood Vessels and Compromise Organ Function in Tumor-Bearing Animals. Cancer Res (2015) 75:2653-62. doi: 10.1158/0008-5472.CAN-14-3299

44. Alfaro C, Teijeira A, Onate C, Perez G, Sanmamed MF, Andueza MP, et al. Tumor-Produced Interleukin-8 Attracts Human Myeloid-Derived Suppressor Cells and Elicits Extrusion of Neutrophil Extracellular Traps (NETs). Clin Cancer Res (2016) 22:3924-36. doi: 10.1158/1078-0432.CCR15-2463

45. Stillie R, Farooq SM, Gordon JR, Stadnyk AW. The Functional Significance Behind Expressing Two IL-8 Receptor Types on PMN. J Leukoc Biol (2009) 86:529-43. doi: $10.1189 / \mathrm{jlb} .0208125$

46. Sanmamed MF, Carranza-Rua O, Alfaro C, Onate C, Martin-Algarra S, Perez G, et al. Serum Interleukin-8 Reflects Tumor Burden and Treatment Response Across Malignancies of Multiple Tissue Origins. Clin Cancer Res (2014) 20:5697-707. doi: 10.1158/1078-0432.CCR-13-3203

47. Teijeira A, Garasa S, Gato M, Alfaro C, Migueliz I, Cirella A, et al. CXCR1 and CXCR2 Chemokine Receptor Agonists Produced by Tumors Induce Neutrophil Extracellular Traps That Interfere With Immune Cytotoxicity. Immunity (2020) 52:856-871 e8. doi: 10.1016/j.immuni.2020.03.001

48. Nie M, Yang L, Bi X, Wang Y, Sun P, Yang H, et al. Neutrophil Extracellular Traps Induced by IL8 Promote Diffuse Large B-Cell Lymphoma Progression via the TLR9 Signaling. Clin Cancer Res (2019) 25:1867-79. doi: 10.1158/ 1078-0432.CCR-18-1226

49. Podaza E, Sabbione F, Risnik D, Borge M, Almejun MB, Colado A, et al. Neutrophils From Chronic Lymphocytic Leukemia Patients Exhibit an Increased Capacity to Release Extracellular Traps (NETs). Cancer Immunol Immunother (2017) 66:77-89. doi: 10.1007/s00262-016-1921-7

50. Zha C, Meng X, Li L, Mi S, Qian D, Li Z, et al. Neutrophil Extracellular Traps Mediate the Crosstalk Between Glioma Progression and the Tumor Microenvironment the HMGB1/RAGE/IL-8 Axis. Cancer Biol Med (2020) 17:154-68. doi: 10.20892/j.issn.2095-3941.2019.0353 
51. Yang L, Liu L, Zhang R, Hong J, Wang Y, Wang J, et al. IL-8 Mediates a Positive Loop Connecting Increased Neutrophil Extracellular Traps (NETs) and Colorectal Cancer Liver Metastasis. J Cancer (2020) 11:4384-96. doi: $10.7150 /$ jca. 44215

52. Cai Z, Zhang M, Boafo Kwantwi L, Bi X, Zhang C, Cheng Z, et al. Breast Cancer Cells Promote Self-Migration by Secreting Interleukin 8 to Induce NET Formation. Gene (2020) 754:144902. doi: 10.1016/j.gene.2020.144902

53. Li Y, Yuan R, Ren T, Yang B, Miao H, Liu L, et al. Role of Sciellin in Gallbladder Cancer Proliferation and Formation of Neutrophil Extracellular Traps. Cell Death Dis (2021) 12:30. doi: 10.1038/s41419-020-03286-Z

54. Guan X, Lu Y, Zhu H, Yu S, Zhao W, Chi X, et al. The Crosstalk Between Cancer Cells and Neutrophils Enhances Hepatocellular Carcinoma Metastasis via Neutrophil Extracellular Traps-Associated Cathepsin G Component: A Potential Therapeutic Target. J Hepatocell Carcinoma (2021) 8:451-65. doi: 10.2147/JHC.S303588

55. Kitagawa Y, Osumi H, Shinozaki E, Ota Y, Nakayama I, Suzuki T, et al. Clinical Utility of Polyethylene Glycol Conjugated Granulocyte ColonyStimulating Factor (PEG-G-CSF) for Preventing Severe Neutropenia in Metastatic Colorectal Cancer Patients Treated With FOLFOXIRI Plus Bevacizumab: A Single-Center Retrospective Study. BMC Cancer (2020) 20:358. doi: 10.1186/s12885-020-06864-8

56. Agarwal S, Lakoma A, Chen Z, Hicks J, Metelitsa LS, Kim ES, et al. G-CSF Promotes Neuroblastoma Tumorigenicity and Metastasis via STAT3Dependent Cancer Stem Cell Activation. Cancer Res (2015) 75:2566-79. doi: 10.1158/0008-5472.CAN-14-2946

57. Liu L, Wu Y, Zhang C, Zhou C, Li Y, Zeng Y, et al. Cancer-Associated Adipocytes-Derived G-CSF Promotes Breast Cancer Malignancy via Stat3 Signaling. J Mol Cell Biol (2020) 12:723-37. doi: 10.1093/jmcb/mjaa016

58. Demers M, Wong SL, Martinod K, Gallant M, Cabral JE, Wang Y, et al. Priming of Neutrophils Toward NETosis Promotes Tumor Growth. Oncoimmunology (2016) 5:e1134073. doi: 10.1080/2162402X.2015.1134073

59. Shang A, Gu C, Zhou C, Yang Y, Chen C, Zeng B, et al. Exosomal KRAS Mutation Promotes the Formation of Tumor-Associated Neutrophil Extracellular Traps and Causes Deterioration of Colorectal Cancer by Inducing IL-8 Expression. Cell Commun Signal (2020) 18:52. doi: 10.1186/ s12964-020-0517-1

60. Leal AC, Mizurini DM, Gomes T, Rochael NC, Saraiva EM, Dias MS, et al. Tumor-Derived Exosomes Induce the Formation of Neutrophil Extracellular Traps: Implications For The Establishment of Cancer-Associated Thrombosis. Sci Rep (2017) 7:6438. doi: 10.1038/s41598-017-06893-7

61. Jablonska E, Garley M, Surazynski A, Grubczak K, Iwaniuk A, Borys J, et al. Neutrophil Extracellular Traps (NETs) Formation Induced by TGF-Beta in Oral Lichen Planus - Possible Implications for the Development of Oral Cancer. Immunobiology (2020) 225:151901. doi: 10.1016/j.imbio.2019.151901

62. Gomes T, Varady CBS, Lourenco AL, Mizurini DM, Rondon AMR, Leal AC, et al. IL-1beta Blockade Attenuates Thrombosis in a Neutrophil Extracellular Trap-Dependent Breast Cancer Model. Front Immunol (2019) 10:2088. doi: 10.3389/fimmu.2019.02088

63. Li Y, Yang Y, Gan T, Zhou J, Hu F, Hao N, et al. Extracellular RNAs From Lung Cancer Cells Activate Epithelial Cells and Induce Neutrophil Extracellular Traps. Int J Oncol (2019) 55:69-80. doi: 10.3892/ijo.2019.4808

64. Singel KL, Grzankowski KS, Anmnh Khan MJ, Grimm AC, D’Auria K, Morrell, et al. Mitochondrial DNA in the Tumour Microenvironment Activates Neutrophils and Is Associated With Worse Outcomes in Patients With Advanced Epithelial Ovarian Cancer. Br J Cancer (2019) 120:207-17. doi: 10.1038/s41416-018-0339-8

65. Zhou J, Yang Y, Gan T, Li Y, Hu F, Hao N, et al. Lung Cancer Cells Release High Mobility Group Box 1 and Promote the Formation of Neutrophil Extracellular Traps. Oncol Lett (2019) 18:181-8. doi: 10.3892/ol.2019.10290

66. Xiao Y, Cong M, Li J, He D, Wu Q, Tian P, et al. Cathepsin C Promotes Breast Cancer Lung Metastasis by Modulating Neutrophil Infiltration and Neutrophil Extracellular Trap Formation. Cance Cell (2021) 39:423-437.e7. doi: 10.1016/j.ccell.2020.12.012

67. Schoeps B, Eckfeld C, Prokopchuk O, Bottcher J, Haussler D, Steiger K, et al. TIMP1 Triggers Neutrophil Extracellular Trap Formation in Pancreatic Cancer. Cancer Res (2021) 81:3568-79. doi: 10.1158/0008-5472.CAN-20-4125

68. Shinde-Jadhav S, Mansure JJ, Rayes RF, Marcq G, Ayoub M, Skowronski R, et al. Role of Neutrophil Extracellular Traps in Radiation Resistance of
Invasive Bladder Cancer. Nat Commun (2021) 12:2776. doi: 10.1038/s41467021-23086-Z

69. Deng J, Kang Y, Cheng CC, Li X, Dai B, Katz MH, et al. Ddr1-Induced Neutrophil Extracellular Traps Drive Pancreatic Cancer Metastasis. JCI Insight (2021) 6:e146133. doi: 10.1172/jci.insight.146133

70. Vito A, El-Sayes N, Mossman K. Hypoxia-Driven Immune Escape in the Tumor Microenvironment. Cells (2020) 9:992. doi: 10.3390/cells9040992

71. Arneth B. Tumor Microenvironment. Med (Kaunas) (2019) 56:15. doi: 10.3390/medicina56010015

72. Zhang Y, Chandra V, Riquelme Sanchez E, Dutta P, Quesada PR, Rakoski A, et al. Interleukin-17-Induced Neutrophil Extracellular Traps Mediate Resistance to Checkpoint Blockade in Pancreatic Cancer. J Exp Med (2020) 217:e20190354. doi: 10.1084/jem.20190354

73. Chrysanthopoulou A, Kambas K, Stakos D, Mitroulis I, Mitsios A, Vidali V, et al. Interferon Lambda1/IL-29 and Inorganic Polyphosphate Are Novel Regulators of Neutrophil-Driven Thromboinflammation. J Pathol (2017) 243:111-22. doi: 10.1002/path.4935

74. Arelaki S, Arampatzioglou A, Kambas K, Sivridis E, Giatromanolaki A, Ritis K. Mast Cells Co-Expressing CD68 and Inorganic Polyphosphate Are Linked With Colorectal Cancer. PloS One (2018) 13:e193089. doi: 10.1371/journal.pone.0193089

75. Schlesinger M. Role of Platelets and Platelet Receptors in Cancer Metastasis. J Hematol Oncol (2018) 11:125. doi: 10.1186/s13045-018-0669-2

76. Abdol Razak N, Elaskalani O, Metharom P. Pancreatic Cancer-Induced Neutrophil Extracellular Traps: A Potential Contributor to Cancer-Associated Thrombosis. Int J Mol Sci (2017) 18:487. doi: 10.3390/ijms18030487

77. Zhang Y, Wang C, Yu M, Zhao X, Du J, Li Y, et al. Neutrophil Extracellular Traps Induced by Activated Platelets Contribute to Procoagulant Activity in Patients With Colorectal Cancer. Thromb Res (2019) 180:87-97. doi: 10.1016/j.thromres.2019.06.005

78. Whittall-García LP, Torres-Ruiz J, Zentella-Dehesa A, Tapia-Rodríguez M, Alcocer-Varela J, Mendez-Huerta N, et al. Neutrophil Extracellular Traps Are a Source of Extracellular HMGB1 in Lupus Nephritis: Associations With Clinical and Histopathological Features. Lupus (2019) 28:1549-57. doi: $10.1177 / 0961203319883936$

79. Munir H, Jones JO, Janowitz T, Hoffmann M, Euler M, Martins CP, et al. Stromal-Driven and Amyloid Beta-Dependent Induction of Neutrophil Extracellular Traps Modulates Tumor Growth. Nat Commun (2021) 12:683. doi: 10.1038/s41467-021-20982-2

80. Guglietta S, Chiavelli A, Zagato E, Krieg C, Gandini S, Ravenda PS, et al. Coagulation Induced by C3aR-Dependent NETosis Drives Protumorigenic Neutrophils During Small Intestinal Tumorigenesis. Nat Commun (2016) 7:11037. doi: 10.1038/ncomms11037

81. Inoue M, Nakashima R, Enomoto M, Koike Y, Zhao X, Yip K, et al. Plasma Redox Imbalance Caused by Albumin Oxidation Promotes LungPredominant NETosis and Pulmonary Cancer Metastasis. Nat Commun (2018) 9:5116. doi: 10.1038/s41467-018-07550-x

82. van der Windt DJ, Sud V, Zhang H, Varley PR, Goswami J, Yazdani HO, et al. Neutrophil Extracellular Traps Promote Inflammation and Development of Hepatocellular Carcinoma in Nonalcoholic Steatohepatitis. Hepatology (2018) 68:1347-60. doi: 10.1002/hep.29914

83. Shrestha S, Kim SY, Yun YJ, Kim JK, Lee JM, Shin M, et al. Retinoic Acid Induces Hypersegmentation and Enhances Cytotoxicity of Neutrophils Against Cancer Cells. Immunol Lett (2017) 182:24-9. doi: 10.1016/ j.imlet.2017.01.001

84. Liu K, Sun E, Lei M, Li L, Gao J, Nian X, et al. BCG-Induced Formation of Neutrophil Extracellular Traps Play an Important Role in Bladder Cancer Treatment. Clin Immunol (2019) 201:4-14. doi: 10.1016/j.clim.2019.02.005

85. Arelaki S, Arampatzioglou A, Kambas K, Papagoras C, Miltiades P, Angelidou I, et al. Gradient Infiltration of Neutrophil Extracellular Traps in Colon Cancer and Evidence for Their Involvement in Tumour Growth PloS One (2016) 11:e0154484. doi: 10.1371/journal.pone.0154484

86. Millrud CR, Kagedal A, Kumlien Georen S, Winqvist O, Uddman R, Razavi R, et al. NET-Producing CD16(high) CD62L(dim) Neutrophils Migrate to Tumor Sites and Predict Improved Survival in Patients With HNSCC. Int $J$ Cancer (2017) 140:2557-67. doi: 10.1002/ijc.30671

87. Muqaku B, Pils D, Mader JC, Aust S, Mangold A, Muqaku L, et al. Neutrophil Extracellular Trap Formation Correlates With Favorable 
Overall Survival in High Grade Ovarian Cancer. Cancers (Basel) (2020) 12:505. doi: $10.3390 /$ cancers 12020505

88. Schedel F, Mayer-Hain S, Pappelbaum KI, Metze D, Stock M, Goerge T, et al. Evidence and Impact of Neutrophil Extracellular Traps in Malignant Melanoma. Pigment Cell Melanoma Res (2020) 33:63-73. doi: 10.1111/pcmr.12818

89. Tohme S, Yazdani HO, Al-Khafaji AB, Chidi AP, Loughran P, Mowen K, et al. Neutrophil Extracellular Traps Promote the Development and Progression of Liver Metastases After Surgical Stress. Cancer Res (2016) 76:1367-80. doi: 10.1158/0008-5472.CAN-15-1591

90. Richardson JJR, Hendrickse C, Gao-Smith F, Thickett DR. Neutrophil Extracellular Trap Production in Patients With Colorectal Cancer In Vitro. Int J Inflam (2017) 2017:4915062. doi: 10.1155/2017/4915062

91. Decker AS, Pylaeva E, Brenzel A, Spyra I, Droege F, Hussain T, et al. Prognostic Role of Blood NETosis in the Progression of Head and Neck Cancer. Cells (2019) 8:946. doi: 10.3390/cells8090946

92. Cedervall J, Dragomir A, Saupe F, Zhang Y, Arnlov J, Larsson E, et al. Pharmacological Targeting of Peptidylarginine Deiminase 4 Prevents Cancer-Associated Kidney Injury in Mice. Oncoimmunology (2017) 6: e1320009. doi: 10.1080/2162402X.2017.1320009

93. Bang OY, Chung JW, Cho YH, Oh MJ, Seo WK, Kim GM, et al. Circulating DNAs, a Marker of Neutrophil Extracellular Traposis and Cancer-Related Stroke: The OASIS-Cancer Study. Stroke (2019) 50:2944-7. doi: 10.1161/ STROKEAHA.119.026373

94. Hell L, Thaler J, Martinod K, Ay C, Posch F, Wagner DD, et al. OC-16 Neutrophil Extracellular Traps and Tissue Factor-Bearing Microvesicles: A Liaison Dangereuse Causing Overt DIC in Cancer Patients? Thromb Res (2016) 140:S174-5. doi: 10.1016/s0049-3848(16)30133-5

95. Yu M, Li T, Li B, Liu Y, Wang L, Zhang J, et al. Phosphatidylserine-Exposing Blood Cells, Microparticles and Neutrophil Extracellular Traps Increase Procoagulant Activity in Patients With Pancreatic Cancer. Thromb Res (2020) 188:5-16. doi: 10.1016/j.thromres.2020.01.025

96. Cedervall J, Dimberg A, Olsson AK. Tumor-Induced Neutrophil Extracellular Traps-Drivers of Systemic Inflammation and Vascular Dysfunction. Oncoimmunology (2016) 5:e1098803. doi: 10.1080/2162402X.2015.1098803

97. Li R, Zou X, Zhu T, Xu H, Li X, Zhu L. Destruction of Neutrophil Extracellular Traps Promotes the Apoptosis and Inhibits the Invasion of Gastric Cancer Cells by Regulating the Expression of Bcl-2, Bax and NFkappaB. Onco Targets Ther (2020) 13:5271-81. doi: 10.2147/OTT.S227331

98. Wang WW, Wu L, Lu W, Chen W, Yan W, Qi C, et al. Lipopolysaccharides Increase the Risk of Colorectal Cancer Recurrence and Metastasis Due to the Induction of Neutrophil Extracellular Traps After Curative Resection. $J$ Cancer Res Clin Oncol (2021) 147:2609-19. doi: 10.1007/s00432-021-03682-8

99. Yang L, Liu Q, Zhang X, Liu X, Zhou B, Chen J, et al. DNA of Neutrophil Extracellular Traps Promotes Cancer Metastasis via CCDC25. Nature (2020) 583:133-8. doi: 10.1038/s41586-020-2394-6

100. Boone BA, Murthy P, Miller-Ocuin J, Doerfler WR, Ellis JT, Liang X, et al. Chloroquine Reduces Hypercoagulability in Pancreatic Cancer Through Inhibition of Neutrophil Extracellular Traps. BMC Cancer (2018) 18:678. doi: 10.1186/s12885-018-4584-2

101. Miller-Ocuin JL, Liang X, Boone BA, Doerfler WR, Singhi AD, Tang D, et al. DNA Released From Neutrophil Extracellular Traps (NETs) Activates Pancreatic Stellate Cells and Enhances Pancreatic Tumor Growth. Oncoimmunology (2019) 8:e1605822. doi: 10.1080/2162402X.2019.1605822

102. Papayannopoulos V. Neutrophil Extracellular Traps in Immunity and Disease. Nat Rev Immunol (2018) 18:134-47. doi: 10.1038/nri.2017.105

103. Yazdani HO, Roy E, Comerci AJ, van der Windt DJ, Zhang H, Huang H, et al. Neutrophil Extracellular Traps Drive Mitochondrial Homeostasis in Tumors to Augment Growth. Cancer Res (2019) 79:5626-39. doi: 10.1158/ 0008-5472.CAN-19-0800

104. Sosa MS, Bragado P, Aguirre-Ghiso JA. Mechanisms of Disseminated Cancer Cell Dormancy: An Awakening Field. Nat Rev Cancer (2014) 14:611-22. doi: $10.1038 /$ nrc3793

105. Albrengues J, Shields MA, Ng D, Park CG, Ambrico A, Poindexter ME, et al. Neutrophil Extracellular Traps Produced During Inflammation Awaken Dormant Cancer Cells in Mice. Science (2018) 361:eaao4227. doi: 10.1126/ science.aao 4227

106. Jung HS, Gu J, Kim JE, Nam Y, Song JW, Kim HK. Cancer Cell-Induced Neutrophil Extracellular Traps Promote Both Hypercoagulability and
Cancer Progression. PloS One (2019) 14:e0216055. doi: 10.1371/ journal.pone. 0216055

107. Jin W, Yin H, Li H, Yu XJ, Xu HX, Liu L. Neutrophil Extracellular DNA Traps Promote Pancreatic Cancer Cells Migration and Invasion by Activating EGFR/ERK Pathway. J Cell Mol Med (2021) 25:5443-56. doi: $10.1111 / j \mathrm{cmm} .16555$

108. Monti M, De Rosa V, Iommelli F, Carriero MV, Terlizzi C, Camerlingo R, et al. Neutrophil Extracellular Traps as an Adhesion Substrate for Different Tumor Cells Expressing RGD-Binding Integrins. Int J Mol Sci (2018) 19:2350. doi: 10.3390/ijms19082350

109. Najmeh S, Cools-Lartigue J, Rayes RF, Gowing S, Vourtzoumis P, Bourdeau F, et al. Neutrophil Extracellular Traps Sequester Circulating Tumor Cells via Beta1-Integrin Mediated Interactions. Int J Cancer (2017) 140:2321-30. doi: 10.1002/ijc. 30635

110. Glogauer JE, Sun CX, Bradley G, Magalhaes MA. Neutrophils Increase Oral Squamous Cell Carcinoma Invasion Through an Invadopodia-Dependent Pathway. Cancer Immunol Res (2015) 3:1218-26. doi: 10.1158/23266066.CIR-15-0017

111. Wang N, Wang Q, Chi J, Xiang F, Lin M, Wang W, et al. Carcinoembryonic Antigen Cell Adhesion Molecule 1 Inhibits the Antitumor Effect of Neutrophils in Tongue Squamous Cell Carcinoma. Cancer Sci (2019) 110:519-29. doi: 10.1111/cas.13909

112. Lu H, Wu B, Ma G, Zheng D, Song R, Huang E, et al. Melatonin Represses Oral Squamous Cell Carcinoma Metastasis by Inhibiting Tumor-Associated Neutrophils. Am J Transl Res (2017) 9:5361-74.

113. Valero C, Zanoni DK, McGill MR, Ganly I, Morris LGT, Quer M, et al. Pretreatment Peripheral Blood Leukocytes Are Independent Predictors of Survival in Oral Cavity Cancer. Cancer (2020) 126:994-1003. doi: 10.1002/cncr.32591

114. Garley M, Jabłońska E, Miltyk W, Grubczak K, Surażyński A, RatajczakWrona W, et al. Cancers Cells in Traps? The Pathways of NETs Formation in Response to OSCC in Humans-A Pilot Study. Cancer Control (2020) 27:1073274820960473. doi: 10.1177/1073274820960473

115. Li B, Liu Y, Hu T, Zhang Y, Zhang C, Li T, et al. Neutrophil Extracellular Traps Enhance Procoagulant Activity in Patients With Oral Squamous Cell Carcinoma. J Cancer Res Clin Oncol (2019) 145:1695-707. doi: 10.1007/ s00432-019-02922-2

116. Garley M, Dziemianczyk-Pakiela D, Grubczak K, Surazynski A, Dabrowska D, Ratajczak-Wrona W, et al. Differences and Similarities in the Phenomenon of NETs Formation in Oral Inflammation and in Oral Squamous Cell Carcinoma. J Cancer (2018) 9:1958-65. doi: 10.7150/jca.24238

117. Cheng FJ, Zhou XJ, Zhao YF, Zhao MH, Zhang H. Human Neutrophil Peptide 1-3, a Component of the Neutrophil Extracellular Trap, as a Potential Biomarker of Lupus Nephritis. Int J Rheum Dis (2015) 18:53340. doi: 10.1111/1756-185x.12433

118. McKeown ST, Lundy FT, Nelson J, Lockhart D, Irwin CR, Cowan CG, et al. The Cytotoxic Effects of Human Neutrophil Peptide-1 (HNP1) and Lactoferrin on Oral Squamous Cell Carcinoma (OSCC) In Vitro. Oral Oncol (2006) 42:685-90. doi: 10.1016/j.oraloncology.2005.11.005

119. Fatehi Hassanabad A, Chehade R, Breadner D, Raphael J. Esophageal Carcinoma: Towards Targeted Therapies. Cell Oncol (Dordr) (2020) 43:195-209. doi: 10.1007/s13402-019-00488-2

120. Tomochika S, Iizuka N, Watanabe Y, Tsutsui M, Takeda S, Yoshino S, et al. Increased Serum Cell-Free DNA Levels in Relation to Inflammation Are Predictive of Distant Metastasis of Esophageal Squamous Cell Carcinoma. Exp Ther Med (2010) 1:89-92. doi: 10.3892/etm_00000016

121. Zhang H, Lv H, Weng M, Wang H, Cata JP, Chen W, et al. Preoperative Leukocytosis is Associated With Increased Tumor-Infiltrating Neutrophil Extracellular Traps and Worse Outcomes in Esophageal Cancer. Ann Transl Med (2020) 8:441. doi: 10.21037/atm.2020.03.190

122. Wada Y, Yoshida K, Tsutani Y, Shigematsu H, Oeda M, Sanada Y, et al. Neutrophil Elastase Induces Cell Proliferation and Migration by the Release of TGF-Alpha, PDGF and VEGF in Esophageal Cell Lines. Oncol Rep (2007) 17:161-7. doi: 10.3892/or.17.1.161

123. Krzystek-Korpacka M, Matusiewicz M, Diakowska D, Grabowski K, Blachut K, Konieczny D, et al. Elevation of Circulating Interleukin-8 Is Related to Lymph Node and Distant Metastases in Esophageal Squamous Cell Carcinomas-Implication for Clinical Evaluation of Cancer Patient. Cytokine (2008) 41:232-9. doi: 10.1016/j.cyto.2007.11.011 
124. Gonzalez-Aparicio M, Alfaro C. Influence of Interleukin-8 and Neutrophil Extracellular Trap (NET) Formation in the Tumor Microenvironment: Is There a Pathogenic Role? J Immunol Res (2019) 2019:6252138. doi: 10.1155/ 2019/6252138

125. Arigami T, Okumura H, Matsumoto M, Uchikado Y, Uenosono Y, Kita Y, et al. Analysis of the Fibrinogen and Neutrophil-Lymphocyte Ratio in Esophageal Squamous Cell Carcinoma: A Promising Blood Marker of Tumor Progression and Prognosis. Med (Baltimore) (2015) 94:e1702. doi: 10.1097/MD.0000000000001702

126. Kawahara Y, Ninomiya I, Fujimura T, Funaki H, Nakagawara H, Takamura H, et al. Prospective Randomized Controlled Study on the Effects of Perioperative Administration of a Neutrophil Elastase Inhibitor to Patients Undergoing VideoAssisted Thoracoscopic Surgery for Thoracic Esophageal Cancer. Dis Esophagus (2010) 23:329-39. doi: 10.1111/j.1442-2050.2009.01010.x

127. Makino H, Kunisaki C, Kosaka T, Akiyama H, Morita S, Endo I. Perioperative Use of a Neutrophil Elastase Inhibitor in Video-Assisted Thoracoscopic Oesophagectomy for Cancer. Br J Surg (2011) 98:975-82. doi: $10.1002 /$ bjs.7499

128. Nishiyama J, Matsuda M, Ando S, Hirasawa M, Suzuki T, Makuuchi H. The Effects of the Early Administration of Sivelestat Sodium, a Selective Neutrophil Elastase Inhibitor, on the Postoperative Course After Radical Surgery for Esophageal Cancer. Surg Today (2012) 42:659-65. doi: 10.1007/ s00595-011-0105-5

129. Olyaee M, Sontag S, Salman W, Schnell T, Mobarhan S, Eiznhamer D, et al. Mucosal Reactive Oxygen Species Production in Oesophagitis and Barrett's Oesophagus. Gut (1995) 37:168-73. doi: 10.1136/gut.37.2.168

130. Kanamaru R, Ohzawa H, Miyato H, Yamaguchi H, Hosoya Y, Lefor AK, et al. Neutrophil Extracellular Traps Generated by Low Density Neutrophils Obtained From Peritoneal Lavage Fluid Mediate Tumor Cell Growth and Attachment. J Vis Exp (2018) 58201. doi: 10.3791/58201

131. Zhu T, Zou X, Yang C, Li L, Wang B, Li R, et al. Neutrophil Extracellular Traps Promote Gastric Cancer Metastasis by Inducing Epithelialmesenchymal Transition. Int J Mol Med (2021) 48:127. doi: 10.3892/ijmm.2021.4960

132. Kanamaru R, Ohzawa H, Miyato H, Matsumoto S, Haruta H, Kurashina K, et al. Low Density Neutrophils (LDN) in Postoperative Abdominal Cavity Assist the Peritoneal Recurrence Through the Production of Neutrophil Extracellular Traps (NETs). Sci Rep (2018) 8:632. doi: 10.1038/s41598-017-19091-2

133. Yang C, Sun W, Cui W, Li X, Yao J, Jia X, et al. Procoagulant Role of Neutrophil Extracellular Traps in Patients With Gastric Cancer. Int J Clin Exp Pathol (2015) 8:14075-86.

134. Zhang Y, Hu Y, Ma C, Sun H, Wei X, Li M, et al. Diagnostic, Therapeutic Predictive, and Prognostic Value of Neutrophil Extracellular Traps in Patients With Gastric Adenocarcinoma. Front Oncol (2020) 10:1036. doi: $10.3389 /$ fonc. 2020.01036

135. Kumagai Y, Ohzawa H, Miyato H, Horie H, Hosoya Y, Lefor AK, et al. Surgical Stress Increases Circulating Low-Density Neutrophils Which May Promote Tumor Recurrence. J Surg Res (2020) 246:52-61. doi: 10.1016/j.jss.2019.08.022

136. Kwon HC, Oh SY, Lee S, Kim SH, Han JY, Koh RY, et al. Plasma Levels of Prothrombin Fragment F1+2, D-Dimer and Prothrombin Time Correlate With Clinical Stage and Lymph Node Metastasis in Operable Gastric Cancer Patients. Jpn J Clin Oncol (2008) 38:2-7. doi: 10.1093/jjco/hym157

137. Ju C, Wen Y, Zhang L, Wang Q, Xue L, Shen J, et al. Neoadjuvant Chemotherapy Based on Abraxane/Human Neutrophils Cytopharmaceuticals With Radiotherapy for Gastric Cancer. Small (2019) 15:e1804191. doi: 10.1002/ smll.201804191

138. Wolpin BM, Mayer RJ. Systemic Treatment of Colorectal Cancer. Gastroenterology (2008) 134:1296-310. doi: 10.1053/j.gastro.2008.02.098

139. Ho AS, Chen CH, Cheng CC, Wang CC, Lin HC, Luo TY, et al. Neutrophil Elastase as a Diagnostic Marker and Therapeutic Target in Colorectal Cancers. Oncotarget (2014) 5:473-80. doi: 10.18632/oncotarget.1631

140. Nicoud IB, Jones CM, Pierce JM, Earl TM, Matrisian LM, Chari RS, et al. Warm Hepatic Ischemia-Reperfusion Promotes Growth of Colorectal Carcinoma Micrometastases in Mouse Liver via Matrix Metalloproteinase-9 Induction. Cancer Res (2007) 67:2720-8. doi: 10.1158/0008-5472.Can-06-3923

141. Dulai PS, Sandborn WJ, Gupta S. Colorectal Cancer and Dysplasia in Inflammatory Bowel Disease: A Review of Disease Epidemiology, Pathophysiology, and Management. Cancer Prev Res (Phila) (2016) 9:88794. doi: 10.1158/1940-6207.CAPR-16-0124
142. Olén O, Erichsen R, Sachs MC, Pedersen L, Halfvarson J, Askling J, et al. Colorectal Cancer in Crohn's Disease: A Scandinavian Population-Based Cohort Study. Lancet Gastroenterol Hepatol (2020) 5:475-84. doi: 10.1016/ s2468-1253(20)30005-4

143. Olén O, Erichsen R, Sachs MC, Pedersen L, Halfvarson J, Askling J, et al. Colorectal Cancer in Ulcerative Colitis: A Scandinavian Population-Based Cohort Study. Lancet (2020) 395:123-31. doi: 10.1016/s0140-6736(19) 32545-0

144. Lehmann T, Schallert K, Vilchez-Vargas R, Benndorf D, Puttker S, Sydor S, et al. Metaproteomics of Fecal Samples of Crohn's Disease and Ulcerative Colitis. J Proteomics (2019) 201:93-103. doi: 10.1016/j.jprot.2019.04.009

145. Bennike TB, Carlsen TG, Ellingsen T, Bonderup OK, Glerup H, Bogsted M, et al. Neutrophil Extracellular Traps in Ulcerative Colitis: A Proteome Analysis of Intestinal Biopsies. Inflammation Bowel Dis (2015) 21:2052-67. doi: 10.1097/MIB.0000000000000460

146. Dinallo V, Marafini I, Di Fusco D, Laudisi F, Franze E, Di Grazia A, et al. Neutrophil Extracellular Traps Sustain Inflammatory Signals in Ulcerative Colitis. J Crohns Colitis (2019) 13:772-84. doi: 10.1093/ecco-jcc/jiy215

147. Fukunaga S, Kuwaki K, Mitsuyama K, Takedatsu H, Yoshioka S, Yamasaki $\mathrm{H}$, et al. Detection of Calprotectin in Inflammatory Bowel Disease: Fecal and Serum Levels and Immunohistochemical Localization. Int J Mol Med (2018) 41:107-18. doi: 10.3892/ijmm.2017.3244

148. Gottlieb Y, Elhasid R, Berger-Achituv S, Brazowski E, Yerushalmy-Feler A, Cohen S. Neutrophil Extracellular Traps in Pediatric Inflammatory Bowel Disease. Pathol Int (2018) 68:517-23. doi: 10.1111/pin.12715

149. Luley K, Noack F, Lehnert H, Homann N. Local Calprotectin Production in Colorectal Cancer and Polyps-Active Neutrophil Recruitment in Carcinogenesis. Int J Colorectal Dis (2011) 26:603-7. doi: 10.1007/s00384-011-1165-0

150. Germann M, Zangger N, Sauvain MO, Sempoux C, Bowler AD, Wirapati P, et al. Neutrophils Suppress Tumor-Infiltrating T Cells in Colon Cancer via Matrix Metalloproteinase-Mediated Activation of TGFbeta. EMBO Mol Med (2020) 12:e10681. doi: 10.15252/emmm.201910681

151. Rayes RF, Vourtzoumis P, Bou Rjeily M, Seth R, Bourdeau F, Giannias B, et al. Neutrophil Extracellular Trap-Associated CEACAM1 as a Putative Therapeutic Target to Prevent Metastatic Progression of Colon Carcinoma. J Immunol (2020) 204:2285-94. doi: 10.4049/jimmunol.1900240

152. Llovet JM, Zucman-Rossi J, Pikarsky E, Sangro B, Schwartz M, Sherman M, et al. Hepatocellular Carcinoma. Nat Rev Dis Primers (2016) 2:16018. doi: 10.1038/nrdp.2016.18

153. Hilscher MB, Shah VH. Neutrophil Extracellular Traps and Liver Disease. Semin Liver Dis (2020) 40:171-9. doi: 10.1055/s-0039-3399562

154. Wang H, Zhang H, Wang Y, Brown ZJ, Xia Y, Huang Z, et al. Regulatory T Cell and Neutrophil Extracellular Trap Interaction Contributes to Carcinogenesis in Non-Alcoholic Steatohepatitis. J Hepatol (2021) 21: S0168-8278. doi: 10.1016/j.jhep.2021.07.032

155. Kaltenmeier CT, Yazdani H, van der Windt D, Molinari M, Geller D, Tsung A, et al. Neutrophil Extracellular Traps as a Novel Biomarker to Predict RecurrenceFree and Overall Survival in Patients With Primary Hepatic Malignancies. HPB (Oxford) (2021) 23:309-20. doi: 10.1016/j.hpb.2020.06.012

156. Seo JD, Gu JY, Jung HS, Kim YJ, Kim HK. Contact System Activation and Neutrophil Extracellular Trap Markers: Risk Factors for Portal Vein Thrombosis in Patients With Hepatocellular Carcinoma. Clin Appl Thromb Hemost (2019) 25:1076029618825310. doi: 10.1177/ 1076029618825310

157. Oklu R, Sheth RA, Wong KHK, Jahromi AH, Albadawi H. Neutrophil Extracellular Traps Are Increased in Cancer Patients But Does Not Associate With Venous Thrombosis. Cardiovasc Diagnosis Ther (2017) 7:S140-9. doi: $10.21037 /$ cdt.2017.08.01

158. Ren J, He J, Zhang H, Xia Y, Hu Z, Loughran P, et al. Platelet TLR4-ERK5 Axis Facilitates NET-Mediated Capturing of Circulating Tumor Cells and Distant Metastasis After Surgical Stress. Cancer Res (2021) 81:2373-85. doi: 10.1158/0008-5472.Can-20-3222

159. Groeneveld DJ, Luyendyk JP. Endothelial Force Awakens a Mechanism of Portal Hypertension: It's a Neutrophil Extracellular Trap! J Thromb Haemost (2019) 17:1201-4. doi: 10.1111/jth.14530

160. Hilscher MB, Sehrawat T, Arab JP, Zeng Z, Gao J, Liu M, et al. Mechanical Stretch Increases Expression of CXCL1 in Liver Sinusoidal Endothelial Cells to Recruit Neutrophils, Generate Sinusoidal Microthombi, and Promote 
Portal Hypertension. Gastroenterology (2019) 157:193-209 e9. doi: 10.1053/ j.gastro.2019.03.013

161. Gong J, Liang YL, Zhou W, Jie Y, Xiao C, Chong Y, et al. Prognostic Value of Neutrophil-to-Lymphocyte Ratio Associated With Prognosis in HBVInfected Patients. J Med Virol (2018) 90:730-5. doi: 10.1002/jmv.25015

162. Hu S, Liu X, Gao Y, Zhou R, Wei M, Dong J, et al. Hepatitis B Virus Inhibits Neutrophil Extracellular Trap Release by Modulating Reactive Oxygen Species Production and Autophagy. J Immunol (2019) 202:805-15. doi: 10.4049/jimmunol.1800871

163. Paillet J, Kroemer G, Pol JG. Immune Contexture of Cholangiocarcinoma. Curr Opin Gastroenterol (2020) 36:70-6. doi: 10.1097/MOG.0000000000000613

164. Gu FM, Gao Q, Shi GM, Zhang X, Wang J, Jiang JH, et al. Intratumoral IL-17 (+) Cells and Neutrophils Show Strong Prognostic Significance in Intrahepatic Cholangiocarcinoma. Ann Surg Oncol (2012) 19:2506-14. doi: 10.1245/s10434-012-2268-8

165. Zhou SL, Dai Z, Zhou ZJ, Chen Q, Wang Z, Xiao YS, et al. CXCL5 Contributes to Tumor Metastasis and Recurrence of Intrahepatic Cholangiocarcinoma by Recruiting Infiltrative Intratumoral Neutrophils. Carcinogenesis (2014) 35:597-605. doi: 10.1093/carcin/bgt397

166. Oto J, Navarro S, Larsen AC, Solmoirago MJ, Plana E, Hervas D, et al. MicroRNAs and Neutrophil Activation Markers Predict Venous Thrombosis in Pancreatic Ductal Adenocarcinoma and Distal Extrahepatic Cholangiocarcinoma. Int J Mol Sci (2020) 21:840. doi: 10.3390/ijms21030840

167. Mady M, Prasai K, Tella SH, Yadav S, Hallemeier CL, Rakshit S, et al. Neutrophil to Lymphocyte Ratio as a Prognostic Marker in Metastatic Gallbladder Cancer. HPB (Oxford) (2020) 22:1490-5. doi: 10.1016/ j.hpb.2020.02.002

168. Tuveson DA, Neoptolemos JP. Understanding Metastasis in Pancreatic Cancer: A Call for New Clinical Approaches. Cell (2012) 148:21-3. doi: 10.1016/ j.cell.2011.12.021

169. Ansari D, Ansari D, Andersson R, Andren-Sandberg A. Pancreatic Cancer and Thromboembolic Disease, 150 Years After Trousseau. Hepatobiliary Surg Nutr (2015) 4:325-35. doi: 10.3978/j.issn.2304-3881.2015.06.08

170. Kruger S, Haas M, Burkl C, Goehring P, Kleespies A, Roeder F, et al. Incidence, Outcome and Risk Stratification Tools for Venous Thromboembolism in Advanced Pancreatic Cancer - A Retrospective Cohort Study. Thromb Res (2017) 157:9-15. doi: 10.1016/j.thromres.2017.06.021

171. Mandala M, Reni M, Cascinu S, Barni S, Floriani I, Cereda S, et al. Venous Thromboembolism Predicts Poor Prognosis in Irresectable Pancreatic Cancer Patients. Ann Oncol (2007) 18:1660-5. doi: 10.1093/annonc/ $\mathrm{mdm} 284$

172. Boone BA, Orlichenko L, Schapiro NE, Loughran P, Gianfrate GC, Ellis JT, et al. The Receptor for Advanced Glycation End Products (RAGE) Enhances Autophagy and Neutrophil Extracellular Traps in Pancreatic Cancer. Cancer Gene Ther (2015) 22:326-34. doi: 10.1038/cgt.2015.21

173. Hisada Y, Mackman N. Update From the Laboratory: Mechanistic Studies of Pathways of Cancer-Associated Venous Thrombosis Using Mouse Models. Hematol-Am Soc Hemat (2019) 2019:182-6. doi: 10.1182/hematology. 2019000025

174. Hisada Y, Grover SP, Maqsood A, Houston R, Ay C, Noubouossie DF, et al. Neutrophils and Neutrophil Extracellular Traps Enhance Venous Thrombosis in Mice Bearing Human Pancreatic Tumors. Haematologica (2020) 105:218-25. doi: 10.3324/haematol.2019.217083

175. Thomas GM, Brill A, Mezouar S, Crescence L, Gallant M, Dubois C, et al. Tissue Factor Expressed by Circulating Cancer Cell-Derived Microparticles Drastically Increases the Incidence of Deep Vein Thrombosis in Mice. J Thromb Haemost (2015) 13:1310-9. doi: 10.1111/jth.13002

176. Kajioka H, Kagawa S, Ito A, Yoshimoto M, Sakamoto S, Kikuchi S, et al. Targeting Neutrophil Extracellular Traps With Thrombomodulin Prevents
Pancreatic Cancer Metastasis. Cancer Lett (2021) 497:1-13. doi: 10.1016/ j.canlet.2020.10.015

177. Takesue S, Ohuchida K, Shinkawa T, Otsubo Y, Matsumoto S, Sagara A, et al. Neutrophil Extracellular Traps Promote Liver Micrometastasis in Pancreatic Ductal Adenocarcinoma via the Activation of Cancerassociated Fibroblasts. Int J Oncol (2020) 56:596-605. doi: 10.3892/ijo.2019.4951

178. Rosales C. Neutrophils at the Crossroads of Innate and Adaptive Immunity. J Leukoc Biol (2020) 108:377-96. doi: 10.1002/JLB.4MIR0220-574RR

179. Lazzaretto B, Fadeel B. Intra- and Extracellular Degradation of Neutrophil Extracellular Traps by Macrophages and Dendritic Cells. J Immunol (2019) 203:2276-90. doi: 10.4049/jimmunol.1800159

180. Donis-Maturano L, Sanchez-Torres LE, Cerbulo-Vazquez A, Chacon-Salinas R, Garcia-Romo GS, Orozco-Uribe MC, et al. Prolonged Exposure to Neutrophil Extracellular Traps can Induce Mitochondrial Damage in Macrophages and Dendritic Cells. Springerplus (2015) 4:161. doi: 10.1186/ s40064-015-0932-8

181. Adrover JM, Nicolas-Avila JA, Hidalgo A. Aging: A Temporal Dimension for Neutrophils. Trends Immunol (2016) 37:334-45. doi: 10.1016/j.it.2016.03.005

182. Hu N, Mora-Jensen H, Theilgaard-Monch K, Doornbos-van der Meer B, Huitema MG, Stegeman CA, et al. Differential Expression of Granulopoiesis Related Genes in Neutrophil Subsets Distinguished by Membrane Expression of CD177. PloS One (2014) 9:e99671. doi: 10.1371/ journal.pone.0099671

183. Zhou G, Yu L, Fang L, Yang W, Yu T, Miao Y, et al. CD177(+) Neutrophils as Functionally Activated Neutrophils Negatively Regulate IBD. Gut (2018) 67:1052-63. doi: 10.1136/gutjnl-2016-313535

184. Mishalian I, Granot Z, Fridlender ZG. The Diversity of Circulating Neutrophils in Cancer. Immunobiology (2017) 222:82-8. doi: 10.1016/ j.imbio.2016.02.001

185. Li M, Lin C, Deng H, Strnad J, Bernabei L, Vogl DT, et al. A Novel Peptidylarginine Deiminase 4 (PAD4) Inhibitor BMS-P5 Blocks Formation of Neutrophil Extracellular Traps and Delays Progression of Multiple Myeloma. Mol Cancer Ther (2020) 19:1530-8. doi: 10.1158/15357163.MCT-19-1020

186. Xia Y, He J, Zhang H, Wang H, Tetz G, Maguire CA, et al. AAV-Mediated Gene Transfer of DNase I in the Liver of Mice With Colorectal Cancer Reduces Liver Metastasis and Restores Local Innate and Adaptive Immune Response. Mol Oncol (2020) 14:2920-35. doi: 10.1002/1878-0261.12787

187. Lazaar AL, Miller BE, Tabberer M, Yonchuk J, Leidy N, Ambery C, et al. Effect of the CXCR2 Antagonist Danirixin on Symptoms and Health Status in COPD. Eur Respir J (2018) 52:1801020. doi: 10.1183/13993003.010202018

Conflict of Interest: The authors declare that the research was conducted in the absence of any commercial or financial relationships that could be construed as a potential conflict of interest.

Publisher's Note: All claims expressed in this article are solely those of the authors and do not necessarily represent those of their affiliated organizations, or those of the publisher, the editors and the reviewers. Any product that may be evaluated in this article, or claim that may be made by its manufacturer, is not guaranteed or endorsed by the publisher.

Copyright (c) 2021 Chen, Han, Qiu, Wang and Zheng. This is an open-access article distributed under the terms of the Creative Commons Attribution License (CC BY). The use, distribution or reproduction in other forums is permitted, provided the original author(s) and the copyright owner(s) are credited and that the original publication in this journal is cited, in accordance with accepted academic practice. No use, distribution or reproduction is permitted which does not comply with these terms. 